\title{
Efficient prediction of thermodynamic properties of quadrupolar fluids from simulation of a coarse-grained model: The case of carbon dioxide
}

\author{
B. M. Mognetti, L. Yelash, P. Virnau, W. Paul, K. Binder* \\ Institut für Physik, Johannes Gutenberg-Universität, \\ Mainz, Staudinger Weg 7, D-55099 Mainz, Germany \\ M. Müller \\ Institut für Theoretische Physik, Georg-August-Universität Göttingen, \\ Friedrich-Hund-Platz 1, D-37077 Göttingen, Germany \\ L. G. MacDowell \\ Dpto. de Quimica Fisica, Facultad de Cc. Quimicas, \\ Universidad Complutense, 28040 Madrid, Spain
}

(Dated: September 4, 2018) 


\begin{abstract}
Monte Carlo simulations are presented for a coarse-grained model of real quadrupolar fluids. Molecules are represented by particles interacting with Lennard-Jones forces plus the thermally averaged quadrupole-quadrupole interaction. The properties discussed include the vapor-liquid coexistence curve, the vapor pressure along coexistence, and the surface tension. The full isotherms are also accessible over a wide range of temperatures and densities. It is shown that the critical parameters (critical temperature, density, and pressure) depend almost linearly on a quadrupolar parameter $q=Q^{* 4} / T^{*}, Q^{*}$ is the reduced quadrupole moment of the molecule and $T^{*}$ the reduced temperature.

The model can be applied to a variety of small quadrupolar molecules. We focus on carbon dioxide as a test case, but consider nitrogen and benzene, too. Experimental critical temperature, density and quadrupolar moment are sufficient to fix the parameters of the model. The resulting agreement with experiments is excellent and marks a significant improvement over approaches which neglect quadrupolar effects. The same coarse-grained model was also applied in the framework of Perturbation Theory (PT) in the Mean Spherical Approximation (MSA). As expected, the latter deviates from the Monte Carlo results in the critical region, but is reasonably accurate at lower temperatures.
\end{abstract}




\section{INTRODUCTION}

Solvents play an essential role in the design and processing of many molecular materials (e.g., oligomers, polymers, etc). In comparison to a melt, the molecular mobility of dissolved substances increases considerably in solution. Not only the flow properties can be controlled easily in solution, but also the phase behavior (and hence the morphology) of the dissolved materials, e.g., by changing the thermodynamic state conditions like temperature, pressure, and concentration.

A particularly important solvent is supercritical carbon dioxide, because the material is inexpensive, nonpoisonous, not reactive, and thermally stable. Hence, its application as a solvent is widespread. $\underline{1}, 2,3$ However, the phase behavior of polymer-solvent systems or other binary fluid mixtures is rather complex in general. When the thermodynamic control parameters temperature $T$, pressure $p$ and solute molar fraction $x$ are varied, various liquid-vapor and fluid-fluid phase equilibria occur, and many different types of (rather complicated) phase diagrams can be observed $\stackrel{4}{\underline{\underline{5}}}$. Even for simple binary fluid mixtures, e.g., carbon dioxide plus short alkanes such as hexadecane, the phase diagram is only known rather incompletely from experiment. $\underline{6}$ These uncertainties also hamper the judgment of the accuracy of the theoretical modeling of such systems. $, \underline{9}, 10,11$ In fact, due to the large control parameter-space that needs to be explored, comprehensive experimental work would be very cumbersome, and a modeling approach seems to be the method of choice. However, the large number of states $(T, p, x)$ that need to be simulated and the complexity of the systems renders a fully chemically realistic all-atom simulation practically impossible. Thus, the construction of a suitable coarse-grained model for such systems containing polymers (or oligomers, respectively) is very desirable. While there is a rich literature on the construction of coarse-grained models for (flexible) polymers, $, \underline{12}, \underline{13}, \underline{14}, \underline{15}, \underline{16}, \underline{17}, 18, \underline{19}$ comparatively little attention has been paid to the question on how a coarse-grained solvent molecule such as $\mathrm{CO}_{2}$ should be described. Iwai et al. $\underline{11}$ and Virnau et al. $\underline{8} \underline{\underline{9}, \underline{10}}$ simply used particles interacting with simple Lennard-Jones forces among themselves and with the beads of the bead-spring chain that represents effective subunits of the polymer. While particles interacting with Lennard-Jones potentials describe noble gases such as liquid argon or neon rather well, it is clear that a "Lennard-Jonesium" is a somewhat unsatisfactory description of a carbon dioxide molecule. While considerable at-

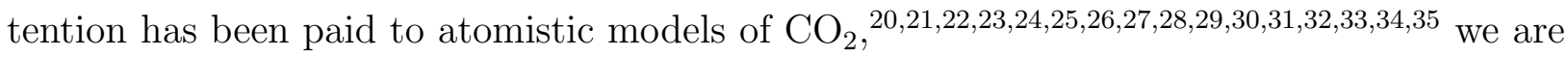


not aware of comprehensive systematic studies of coarse-grained models for this molecular fluid. With respect to the atomistic models for $\mathrm{CO}_{2}$, we note that there is no consensus in the literature on a unique form of the interaction potential and its parameters. Starting from the Murthy-Singer-McDonald (MSM) model, ${ }^{20}$ several potentials have been proposed (for a recent comparison see Ref. 29). In Ref. 27, two variants of the Elementary Physical Model (EPM) force field were suggested, that yielded critical temperatures of $T_{c}=313.4 \pm 0.7 \mathrm{~K}$ and $312.8 \pm 3.0 \mathrm{~K}$, respectively, while the experimental value is $T_{c}=304.2 \mathrm{~K} . \underline{36} \mathrm{In}$ view of this $3 \%$ discrepancy between the atomistic models and the experiment, it was suggested 27 to use the experimental critical temperature and rescale the energy parameters of the model to reproduce the correct value of the critical temperature (EPM2). In fact, Virnau et al. $\underline{8}, \underline{9}, 10$, using a simple "Lennard-Jonesium" to model $\mathrm{CO}_{2}$, fixed the Lennard-Jones parameters to match both the critical temperature $T_{c}$ and the critical density $\rho_{c}$. Since the atomistic models underestimate the critical density (yielding $27 \quad 453.7 \pm 4.3 \mathrm{~kg} / \mathrm{m}^{3}$ or $449 \pm 16 \mathrm{~kg} / \mathrm{m}^{3}$ instead of the experimental value ${ }^{36} \rho_{c}=468.0 \mathrm{~kg} / \mathrm{m}^{3}$ ), they also require a corresponding rescaling of the interaction range parameters. Hence, EPM2 needs the same input from the experimental critical data as the coarse-grained model of Virnau et al. $\underline{8}, \underline{9}, \underline{10}$. For the resulting model, the coexistence densities predicted for the liquid branch in the temperature region $230 \mathrm{~K} \leq T \leq 280 \mathrm{~K}$ deviate distinctly less from the experimental results ${ }^{36}$ than the corresponding results of the coarse-grained model. $\underline{8,10}$

As indicated above, the main interest for obtaining an accurate coarse-grained model for $\mathrm{CO}_{2}$ is its potential application in multicomponent systems, e.g., polymer solutions in which $\mathrm{CO}_{2}$ acts as a solvent. $\stackrel{8,10}{-}$ For such systems also many attempts were undertaken to derive approximate analytical equations of states (e.g., Refs. 37,38) and it is, of course, also highly desirable to validate such equation of state theories by simulations. However, the coarsegrained model for $\mathrm{CO}_{2}$ of Virnau et al. $\stackrel{8,10}{ }$, when combined with a suitable coarse-grained model for the alkanes, required rather large deviations from the simple Lorentz-Berthelot mixing rules to account for the available experimental data. $\frac{6.7}{}$ Most likely, the somewhat oversimplified $\mathrm{CO}_{2}$ model is responsible for most of these deviations. Approximating $\mathrm{CO}_{2}$ as a Lennard-Jones particle without considering its rather large quadrupolar moment $(|Q|=4.3$ $\mathrm{DA}$ ) is probably not sufficient - the unit D (Debye) equals $10^{-18}$ in CGS units which are adopted throughout the manuscript.

In the present paper we explore a slightly more involved coarse-grained model for $\mathrm{CO}_{2}, \underline{39}, \underline{40}$ 
The molecule is still described as a Lennard-Jones particle, but we also include the experimentally known quadrupole moment as an input parameter, together with critical temperature and critical density. A precondition for the usefulness of coarse-grained models is that simulation codes execute very fast. The angular-dependent quadrupole-quadrupole interaction requires significant computational resources which would be a serious drawback to such a model. However, compared to the Lennard-Jones forces, the quadrupolar interaction is still a rather weak perturbation. Therefore, we apply one further approximation: $\underline{39}$ the angular dependence is averaged over in a second order thermodynamic perturbation calculation. Thus, an effective isotropic potential is obtained. Rather encouraging results using such an approximation have been reported in the literature. $\underline{\underline{40}}$ Müller and Gelb $\underline{\underline{40}}$ estimate coexistence curves from non-equilibrium molecular dynamics (NEMD) simulations of temperature quenches from the one-phase region into the two-phase region, where one then waits until the system has phase separated into the two coexisting phases. $\underline{\underline{41}} \underline{\underline{42}}$ In this

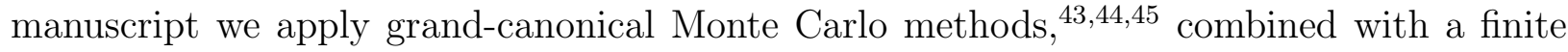
size scaling $55,46,47$ analysis. This allows us to locate precisely the critical point of the model. Note that a direct estimation of the critical point from the simulation is difficult if either Gibbs ensemble techniques $\underline{48}, \underline{49}$ or the temperature quench technique $\underline{40} \underline{\underline{41}}$ are applied. In these cases, one relies on a fit of the coexistence data to a suitable power law extrapolation. With the present techniques one can obtain the critical properties very accurately. This precision is required because the critical properties are used to gauge the Lennard-Jones parameters of the model.

In Sec. II, we give a more detailed description of our model and simulation techniques. Sec. III describes our results for carbon dioxide and compares them to previous approaches. Sec. IV discusses the application of the model to other quadrupolar fluids, namely nitrogen and benzene. Sec. V describes the application of first order perturbation theory in the mean spherical approximation (PT-MSA) to precisely the same model which was used in the simulation, thus allowing a meaningful comparison. Finally, Sec. VI concludes the discussion and gives an outlook on future work. 


\section{MODEL AND SIMULATION TECHNIQUE}

\section{A. Choice of Model}

Our model system consists of neutral spherical particles which carry a quadrupolar moment $Q$ and interact with each other both via the Lennard-Jones potential

$$
U_{i j}^{L J}=4 \varepsilon\left[\left(\frac{\sigma}{r_{i j}}\right)^{12}-\left(\frac{\sigma}{r_{i j}}\right)^{6}\right]
$$

and the quadrupole-quadrupole interaction ${ }^{50}$

$$
U_{i j}^{Q Q}=\frac{3 Q^{2}}{4 r_{i j}^{5}} f^{Q Q}\left(\theta_{i}, \theta_{j}, \phi_{i j}\right) .
$$

The angle-dependent part is given by:

$$
\begin{array}{r}
f^{Q Q}=1-5 \cos ^{2} \theta_{i}-5 \cos ^{2} \theta_{j}+17 \cos ^{2} \theta_{i} \cos ^{2} \theta_{j} \\
+2 \sin ^{2} \theta_{i} \sin ^{2} \theta_{j} \cos ^{2}\left(\phi_{i}-\phi_{j}\right) \\
-16 \sin \theta_{i} \cos \theta_{i} \sin \theta_{j} \cos \theta_{j} \cos \left(\phi_{i}-\phi_{j}\right)
\end{array}
$$

In Eqs. (11, 2), $r_{i j}=\left|\vec{r}_{i}-\vec{r}_{j}\right|$ is the distance between molecules at sites $\vec{r}_{i}, \vec{r}_{j}$, while $\left(\theta_{i}, \phi_{i}\right)$ are the polar angles characterizing the mutual orientations of the (linear) molecules ( $\theta_{i}$ are the angles between the axis joining the two molecules and the quadrupole vectors of the molecules; $\phi_{i}$ are the rotational orientations of the quadrupole vectors relative to the joining axis). In Eq. (1), $\varepsilon$ and $\sigma$ set the scales of energy and distance for the Lennard-Jones (LJ) interaction, respectively.

The angular-dependent part of the potential (Eqs. (2), (3) ) slows down the speed of the algorithm considerably. Therefore, following Ref. 39, we average over the angles of the quadrupolar potential to create an effective isotropic representation. More precisely, one

expands the Boltzmann factor $\exp \left(-\beta U_{i j}^{Q Q}\right),\left(\beta=\left(k_{B} T\right)^{-1}\right)$, in a Taylor series to second order in $\beta$. After taking averages over the angles, the following temperature-dependent isotropic potential is obtained:

$$
U_{i j}^{I Q Q}=-\frac{7 \beta}{5} \frac{Q^{4}}{r_{i j}^{10}}
$$


For the potentials of Eq. (1) to Eq. (44) one can employ the standard procedure ${ }^{43}, 44$ of cutting and shifting to zero at a cutoff distance $r_{i j}=r_{c}=2 \sqrt[6]{2} \sigma$ typically applied to Lennard-Jones systems. The total potential then reads

$$
U\left(r_{i j}\right)=\left\{\begin{array}{l}
4 \varepsilon\left[\left(\frac{\sigma}{r_{i j}}\right)^{12}-\left(\frac{\sigma}{r_{i j}}\right)^{6}-\frac{7}{20} q\left(\frac{\sigma}{r_{i j}}\right)^{10}+S\right], \quad r \leq r_{c} . \\
0 \quad, \quad r \geq r_{c} .
\end{array}\right.
$$

The reduced quadrupolar interaction parameter is defined as

$$
q=\frac{Q^{4}}{\varepsilon \sigma^{10} k_{B} T}=q_{c} \frac{T_{c}}{T}, \quad q_{c}=q\left(T_{c}\right)
$$

$q_{c}$ and $T_{c}$ are the values of the reduced quadrupole parameter and temperature at the critical point. $S$ shifts the cut potential to zero at $r_{i j}=r_{c}$, so that $U\left(r_{i j}\right)$ is continuous everywhere:

$$
S=\frac{127}{16384}+\frac{7}{5} \frac{q}{256}
$$

Note that Eq. (6) is given in CGS units. In SI units, there is an additional factor $\left(4 \pi \varepsilon_{0}\right)^{-2}$. It is clear that $U\left(r_{i j}\right)$ is explicitly temperature-dependent because $q$ and $S$ are temperaturedependent. Hence, special care needs to be exerted when temperature derivatives are taken. For instance, the fluctuation relation linking the specific heat to the fluctuations of the potential energy no longer holds for Eq. (5). We also note that Eq. (5) differs from the potential obtained when one cuts off Eqs. (12) at $r_{i j}=r_{c}$. Indeed, continuity of $U_{i j}^{Q Q}$ would require an orientation-dependent shift of the potential. It is also well-known ${ }^{51}$ that the relation between the critical temperature of a fluid and the energy scale $\varepsilon$ of the LJ interaction depends rather strongly on the cutoff $r_{c}$. Our choice of a rather small value for the cutoff is mainly motivated by the desire to have a very fast simulation algorithm, but larger cutoffs will lead to very similar results. As we will demonstrate later, differences in the phase diagram almost disappear when simulation data are rescaled to match the experimental critical point for different $r_{c}$. A further motivation for this choice of the cutoff is that for $q=0$ our model reduces to that of Refs. 8, 10 .

Our strategy will be to compute the critical temperature $T_{c}\left(q_{c}\right)$ and the critical density $\rho_{c}\left(q_{c}\right)$ from the simulation, using the potential from Eq. (5 5 ). Following previous work ${ }^{8,10}$, $\varepsilon$ and $\sigma$ are determined by the condition that these critical parameters match precisely their experimental counterparts. In the following, $T^{*}$ and $\rho^{*}$ will refer to temperatures 
and densities (and other quantities that will be introduced with "*") expressed in units of $\varepsilon\left(q_{c}\right), \sigma\left(q_{c}\right)$ and $M_{\mathrm{Mol}}$, the molar mass of the fluid. We need to consider that the parameter $q_{c}$, Eq. (6), depends itself on $\varepsilon$ and $\sigma$, and not only on the (given) experimental value for Q. This difficulty is related to the quadrupolar interaction in Eq. (5), which shifts the critical point in the $(T, \rho)$ plane relative to its position for $Q=0$. Even if one is only interested in a single choice of $Q$, a simulation of a single model system (i.e., one choice of $\varepsilon, \sigma$ and $Q$ or $q$, respectively) is never sufficient to deal with this problem. However, this puzzle can be solved by determining the critical lines $T_{c}^{*}\left(q_{c}\right)$ and $\rho_{c}^{*}\left(q_{c}\right)$ as a function of the (dimensionless) parameter $q_{c}$. Fig. 1 shows the results of this calculation and demonstrates that both $T_{c}^{*}\left(q_{c}\right) / T_{c}^{*}(0)$ and $\rho_{c}^{*}\left(q_{c}\right) / \rho_{c}^{*}(0)$ are very smooth functions of $q_{c}$. These curves are almost linear, so recording a few (altogether 9 ) choices of nonzero $q_{c}$ was sufficient to obtain good accuracy. In the range $0 \leq q_{c} \leq 0.5$, the critical temperature increases by almost $30 \%$ while the critical density increases by about $10 \%$.

Having determined $T_{c}^{*}\left(q_{c}\right)$ and $\rho_{c}^{*}\left(q_{c}\right)$, one can compute easily $\varepsilon\left(q_{c}\right)$ and $\sigma\left(q_{c}\right)$ such that the model corresponds to a specific experimental system $T_{c, \exp }$ and $\rho_{c \text {,exp }}$. Eq. (6) must hold together with

$$
\varepsilon\left(q_{c}\right)=k_{B} T_{c, \exp } / T_{c}^{*}\left(q_{c}\right), \quad \sigma^{3}\left(q_{c}\right)=\left[\frac{\rho_{c}^{*}\left(q_{c}\right) M_{\mathrm{Mol}}}{\rho_{c, \exp } N_{A}}\right] .
$$

Here $M_{\mathrm{Mol}}$ is the molar mass of the simple molecule and $N_{A}$ Avogadro's number. These equations are solved by a simple iteration procedure, using the following fit functions representing the data of Fig. 1,

$$
\begin{aligned}
T_{c}^{*}\left(q_{c}\right) / T_{c}^{*}(0) & =1+0.46111 q_{c}+0.17571 q_{c}^{2} \\
\rho_{c}^{*}\left(q_{c}\right) / \rho_{c}^{*}(0) & =1+0.19298 q_{c}
\end{aligned}
$$

where $\mathrm{T}_{c}^{*}\left(q_{c}=0\right)=0.99821$ and $\rho_{c}^{*}\left(q_{c}=0\right)=0.32276$. Appendix $\mathrm{B}$ explains in detail how simulation parameters are derived from experimental data. We note that the limiting factor for the accuracy of our procedure is not at all the limited accuracy of Eqs. (9a, 9b), but rather the uncertainty with which the physical quadrupole moment $Q$ of the molecule, needed as an input to Eq. (6) , is known. Considering $\mathrm{CO}_{2}$ as an example, we take $Q=(-4.3 \pm 0.2)$ $\mathrm{DA}$. However, since $Q$ is raised to the fourth power in Eq. (6]), the $5 \%$ uncertainty in $Q$ 
becomes a $30 \%$ uncertainty in the reduced simulation parameter $q$. For $Q=-4.3 \mathrm{D} \AA$, we obtain

$$
q_{c}=0.387, \quad \varepsilon=3.491 \times 10^{-21} J, \quad \sigma=3.785 \AA
$$

The uncertainty in $Q$ would actually allow for a range $0.32<q_{c}<0.47$ with corresponding changes of $\varepsilon$ and $\sigma$. In view of these uncertainties, one could not hope for a perfect agreement between the simulation results (for other quantities rather than $\rho_{c}$ and $T_{c}$ ) and experiment, even if the form of the coarse-grained potential, Eqs. (5-7), were perfectly accurate.

Already at this point, we note that nothing in the model (Eqs. (5)-(17)) is specific to $\mathrm{CO}_{2}$. Hence, Fig. 1 (or Eqs. (9a, 9b), respectively) can be used for modeling other quadrupolar fluids, too. This fact will be taken up in Sec. IV and Appendix B. We also note that $\varepsilon$ and $\sigma$ are independent of the state of the system once they are fixed. $q$, however, is given by $q=q_{c} \cdot T_{c} / T$ (according to Eq. (6) ), which needs to be considered when coexistence curve and interfacial tension are calculated.

\section{B. Comments on the Simulation Technique}

In this section we comment briefly on the Monte Carlo simulation techniques which are required for the computation of Fig. 1 and other physical properties. As in previous work, $\underline{8}, 10$ extensive simulations were undertaken in the $\mu V T$ ensemble, where the box volume $V=L^{3}$, the chemical potential $\mu$ of the particles and the temperature are fixed. The particle

number fluctuates, since the elementary Monte Carlo move consists of random insertions or deletions of particles. Thus, long wavelength fluctuations of the density are equilibrated easily. In contrast, Molecular Dynamics or canonical ensemble Monte Carlo methods that conserve the particle number in the system suffer from a slow equilibration of long wavelength density fluctuations ("hydrodynamic slowing down" $\underline{\underline{4}}$ ). The temperature quench simulations encounter the additional difficulty that vapor-liquid interfaces extending throughout the simulation box are formed. Such interfaces are notoriously slowly relaxing and strongly fluctuating objects and thus avoided in Gibbs ensemble techniques $\underline{\underline{48}} \underline{\underline{49}}$

For the sake of efficiency, histogram extrapolation techniques are used. In a typical MC run, the particle number $n$ and the total energy $E$ are recorded at regular intervals. The resulting distribution $P_{\mu, T}(n, E)$ can then be extrapolated to neighboring values of $\mu^{\prime}$ and 
$T^{\prime}$ using the following expression $\underline{52}$

$$
P_{\mu^{\prime}, T^{\prime}}(n, E)=\frac{1}{\mathcal{N}} P_{\mu, T}(n, E) \exp \left[\left(\frac{\mu^{\prime}}{T^{\prime}}-\frac{\mu}{T}\right) n-\left(\frac{1}{T^{\prime}}-\frac{1}{T}\right) E\right]
$$

with $\mathcal{N}$ being a normalization constant. Here, we have assumed that $q_{c}$ remains constant. Extrapolations at constant $Q$ would require an additional reweighting factor related to the temperature-dependence of the potential (Eq. (5)). Of course, Eq. (11) is only accurate when $P_{\mu, T}(n, E)$ and $P_{\mu^{\prime}, T^{\prime}}(n, E)$ overlap strongly. Nevertheless, reweighting is very useful for $\mu$ near $\mu_{\text {coex }}(T)$, where two-phase coexistence between vapor and liquid occurs. In this region, $P_{\mu, T}(n)=\int d E P_{\mu, T}(n, E)$ has a two-peak structure: one peak occurs at $\rho_{\text {coex }}^{(1)} \approx n / V$, the vapor density at coexistence, the other peak at $\rho_{\text {coex }}^{(2)} \approx n / V$, the liquid density at coexistence. For $\mu=\mu_{\text {coex }}(T)$, the areas underneath both peaks are equal ("equal area rule" $\underline{53}, \underline{54}$ ), but unfortunately $\mu_{\text {coex }}(T)$ is not known beforehand. However, if one has $P_{\mu, \mathrm{T}}(n)$, for some $\mu$ close enough to $\mu_{\text {coex }}(T)$, one can try to reweight the data according to Eq. (11) with no additional simulation effort. In this way, the coexistence curve can be located precisely. The corresponding pressure is computed from the virial equation. All these procedures have already been applied in previous work for $q_{c}=0$. For more details the reader is referred to Refs. 8, 10.

Following a path along $\mu=\mu_{\text {coex }}(T)$ in the $(\mu, T)$ plane and recording moments of the density distribution, we calculate $2^{\text {nd }}$ and $4^{\text {th }}$ order cumulants

$$
U_{2}=\left\langle M^{2}\right\rangle /\langle|M|\rangle^{2} \quad, \quad U_{4}=\left\langle M^{4}\right\rangle /\left\langle M^{2}\right\rangle^{2} \quad, \quad M \equiv \rho-\langle\rho\rangle .
$$

Reasonably accurate estimates for $T_{c}$ can be obtained from the intersection point of either $U_{2}(T)$ or $U_{4}(T)$ for different $L$. The justification of this simple recipe follows from the theory of finite size scaling. $\underline{43,44,45,46,47} \mathrm{Fig}$. 2 shows that $T_{c}$ can be determined with a relative accuracy of about $3 / 10^{3}$ with moderate computational effort. The lack of perfect intersections in the size range $9 \sigma \leq L \leq 13.5 \sigma$ indicates that the asymptotic region of finite size scaling has not been reached yet, and corrections to finite size scaling are still present. However, the estimate $k_{B} T_{c} / \varepsilon=1.152 \pm 0.003$ is clearly accurate enough for our present purposes. Note that the simple analysis presented in Fig. 2 ignores "field mixing"effects $\underline{45}$ between density and energy per particle. Of course, for a high precision study of critical exponents and critical amplitudes, more sophisticated finite size scaling methods are 
available, ${ }^{55}$ but this is beyond the scope of the present investigation.

For temperatures distinctly below $T_{c}$, the double-peak distribution $P_{\mu, T}(n)$ exhibits a deep minimum for densities $\rho$ in between the two coexisting phases $\rho_{\text {coex }}^{(1)}[\operatorname{vapor}]$ and $\rho_{\text {coex }}^{(2)}[$ liquid].$\underline{56}$ Consequently, a system starting with a low vapor-like density would hardly ever make the transition to the liquid-like state or vice versa. Hence, the relative weights of the two phases would not be sampled correctly. This difficulty is overcome by biased sampling methods that "drive" the system through the coexistence region such as "multicanonical sampling", 57 "Wang-Landau-sampling" $\underline{58}$ or "successive umbrella sampling" $\underline{59}$ which has been used in this work. In the simplest implementation, the algorithm is constrained to sample configurations with only two particles $n \in(0,1)$ in the beginning, and $(1,2) \cdots(n-1, n)$ later on, spanning the relevant range of densities. The probability distribution can then be calculated recursively:

$$
\frac{P(n)}{P(0)}=H_{1,0} H_{2,1} \cdots H_{n, n-1}
$$

with $H_{j, j-1}$ being the frequency of occurrence of the $j^{\text {th }}$ particle over the frequency of occurrence of the $(j-1)^{\text {th }}$ particle in the sampling of the $(j-1, j)$ window. For a more detailed description of this method and its extension we refer to Virnau et al. $\underline{8,10,59}$. Biased grand canonical methods have the additional advantage that the minimum in $P_{\mu, T}(n)$ at densities near the density of the rectilinear diameter $\rho_{d}(T)$

$$
\rho_{d}(T)=\left(\rho_{\text {coex }}^{(1)}+\rho_{\text {coex }}^{(2)}\right) / 2
$$

is also sampled rather accurately. This minimum $43,44,45,56$ corresponds to a free energy barrier $\Delta F \approx 2 \gamma(T) L^{2}$ which arises from the formation of two (planar) vapor-liquid interfaces of area $L^{2}$, each connected with itself via periodic boundary conditions. In this expression, $\gamma(T)$ is the vapor-liquid interfacial tension. For $\rho$ near $\rho_{d}(T)$, the system is in a state of two-phase coexistence, a slab-like liquid domain is separated from the vapor via those interfaces. Coexisting gas and liquid phases have the same free energy. Therefore, $\Delta F$ is the free energy of the interface. It has been amply verified for a variety of systems $\underline{56}, 60,61,62,63,64$ that the relation 56

$$
P_{\mu, T}\left(n_{d}\right) / P_{\mu, T}\left(n_{\text {coex }}\right) \propto \exp \left[-2 \gamma(T) L^{2} / k_{B} T\right]
$$

(where $n_{d}=\rho_{d}(T) L^{3}$ and $n_{\text {coex }}=\rho_{\text {coex }}^{(1,2)} L^{3}$ ) is a valid description of the simulation results, 
and can be used to extract rather accurate estimates for $\gamma(T)$.

Close to $T_{c}$ the estimates for $\rho_{\mathrm{coex}}^{(1)}, \rho_{\mathrm{coex}}^{(2)}, \rho_{d}$, and $\gamma(T)$ suffer from systematic finite size effects. It turns out, however, that the finite size effects for $\rho_{d}$ are numerically rather small. Therefore, the critical density $\rho_{c}$ can be estimated from $\rho_{c}=\rho_{d}\left(T_{c}\right)$ with Eq. (14). $\rho_{\text {coex }}^{(1)}$ and $\rho_{\text {coex }}^{(2)}$ are just the peak values of the density resulting from the equal area rule at $T_{c}$. (We note that $\rho_{\text {coex }}^{(2)}\left(T_{c}\right)>\rho_{\text {coex }}^{(1)}\left(T_{c}\right)$ for any finite $L$. The peak values only merge into a single point $\rho_{c}$ at $\mathrm{T}_{c}$ in the thermodynamic limit.)

The behavior of the density near the critical point can then be obtained, too. In the critical region the critical exponent $\beta$ has to take the value $\beta=0.325$ of the Ising model universality class 65

$$
\begin{aligned}
& \rho_{\text {coex }}^{(1)}-\rho_{d}(T)=-\hat{B}\left(1-T / T_{c}\right)^{\beta} \\
& \rho_{\text {coex }}^{(2)}-\rho_{d}(T)=+\hat{B}\left(1-T / T_{c}\right)^{\beta} .
\end{aligned}
$$

Here, the critical amplitude $\hat{B}$ can be estimated by fitting the actual simulation data in the range $0.02 \leq 1-T / T_{c} \leq 0.1$ to Eq. (16). Note that the left boundary of this interval is chosen such that for the typical linear dimensions, finite size effects on the peak position estimates for $\rho_{\text {coex }}^{(1)}, \rho_{\text {coex }}^{(2)}$ are still very small. The right boundary of the interval is chosen in order to justify the neglect of correction terms to the leading term written in Eq. (16) which only describes the asymptotic behavior in the limit 65 $1-T / T_{c} \rightarrow 0$.

Our data for the coexistence curve and interfacial tension were derived from an elongated box $L \times L \times 2 L$ with size $L=9 \sigma$ and $L=6.74 \sigma$ (the latter only very far from the critical point). The critical points (Figs. 1, 2) were computed using cubic boxes of size $9 \sigma$ and 11.3 $\sigma$. In a few cases, a larger box $L=13.5 \sigma$ was implemented to check the finite size effects. After coexistence densities were determined, simulations at coexistence gas density were carried out in the NVT ensemble to obtain the coexistence pressure from the standard virial expression. 


\section{NUMERICAL RESULTS FOR CARBON DIOXIDE: COMPARISON WITH EXPERIMENT AND SIMULATIONS OF ATOMISTIC MODELS}

Figs. 3.5 present the coexistence curve, the vapor pressure at coexistence, and the interfacial tension as a function of temperature, and compare them to pertinent experimental data. ${ }^{66}$ If quadrupolar interactions are neglected $\left(q_{c}=0\right)$, a distinct discrepancy between the experimental data and the simulations can be observed for the liquid branch of the coexistence curve. $\stackrel{8}{10}^{10}$ Agreement with experiments improves considerably for the isotropic quadrupolar model. A value of $q_{c}=0.387$ was used which corresponds to the experimental value of the $\mathrm{CO}_{2}$ quadrupolar moment $|Q|=4.3 \mathrm{DA}$ (Eq. (10) ) as discussed above. It is also very gratifying that both coexistence pressure (Fig. 4) and interfacial tension (Fig. 51) are in almost perfect quantitative agreement with experimental data, although for these quantities there is no adjustable parameter available whatsoever. In particular, the interfacial tension for $q_{c}=0$ deviates from the experimental data rather distinctly, while for $q_{c}=0.387$ there is excellent agreement.

A small but systematic discrepancy is still present for the liquid branch of the coexistence curve (Fig. 3). Hence, we have also tried to take $q_{c}$ as an adjustable parameter to optimize the agreement between the simulated coexistence curve and the experiments. The rationale for doing so is twofold: first, there is a considerable uncertainty in the experimental value for $Q$, leading to a $30 \%$ uncertainty in $q_{c}$ - it is not even clear that the value of $Q$ for $\mathrm{CO}_{2}$ in the vapor phase and in the liquid are exactly the same. Secondly, it might be better to choose an effective value for $Q$ because our spherically symmetric model (Eq. (5)) is a rather incomplete description for the interactions between elongated $\mathrm{CO}_{2}$ molecules. In principle, the systematic coarse-graining of a chemically realistic model could lead to some effective value for $Q$, which is larger than the experimental one.

Thus, Figs. 3 35 also include some simulation results for a second choice of $q_{c}$, namely $q_{c}=0.470$. Fig. 3 shows that now the agreement between simulation and experiment for the liquid branch of the coexistence curve is better than for $q_{c}=0.387$, but for the vapor branch it is slightly worse. The same slight deterioration of the agreement can also be observed for the coexistence pressure (Fig. 4) and the interface tension (Fig. 5). We conclude that an absolutely perfect agreement between any simplified model, such as Eq. (5), and a real system simply cannot be expected. Some uncertainty about the optimum choice of the parameters 
of such a coarse-grained model is simply inevitable. Actually, the level of agreement between experiment and our model is very good for both choices of $q_{c}$. This is gratifying, since the model will serve as an excellent starting point for the coarse-grained modeling of various polymer solutions containing $\mathrm{CO}_{2}$ as a solvent.

A model of the type of Eq. (5) (named isotropic multipolar or IMP) was also used in Ref. 40,42 and the vapor-liquid coexistence curve of $\mathrm{CO}_{2}$ was determined with temperature quench MD techniques. $\stackrel{41}{\underline{*}}$ The simulation results of Ref. 42 are reported in Fig. 3 (see o), too. Although large systems were used, error bars in the determination of the coexisting densities using NEMD are large in comparison with ours as discussed above. (Errors for our simulations are smaller than the size of the symbols and therefore not shown in Figs. 3. 5.) We also note that Ref. 42 uses Lennard-Jones parameters that differ significantly from ours, namely $\varepsilon / k_{B}=215.0 \mathrm{~K}$ and $\sigma=3.748 \AA$ while we use $\varepsilon / k_{B}=252.8$ and $\sigma=3.785 \AA$ for $|Q|=4.3 \mathrm{DA}$. This is mainly related to the larger cutoff radius of $4 \sigma$ used in Ref. 42, which increases the critical temperature. Our agreement with experimental results (i.e., coexistence curve Fig. [3, coexistence pressure Fig. 4 and isobar Fig. 8) is, however, clearly very good because our grandcanonical simulations allows for a very precise determination of the critical point.

Let us ask how our simulation results for the coarse-grained model compare to the results obtained for atomistic models of $\mathrm{CO}_{2}$. Figs. 6, 7 and 8 present such comparisons for the coexistence densities and pressures with some results available in literature. The EPM model $^{27}$ (denoted by + in Figs. 6] and 7) overestimates the vapor density at coexistence and underestimates the coexistence pressure systematically, while the liquid densities are underestimated only for $T \leq 260 \mathrm{~K}$. For $T \geq 280 \mathrm{~K}$, the liquid densities of the atomistic simulation are too large due to the overestimation of $T_{c}$. When the atomistic model is rescaled (EPM2) 27 so that the critical temperature and density are matched (denoted by $\triangleleft$ in Figs. 6 and 7), the agreement between the model calculation and experiment is almost as good as for our coarse-grained model. However, the rescaled data for the coexistence pressure are slightly but systematically too large. The coexistence line for the EPM2 model has also been obtained in Ref. 28, in agreement with the previous work. ${ }^{27}$ In Fig. 8 we include simulation results of Ref. 28 for the EPM2 model for the supercritical isobar (200 bar). The both models work very good in the supercritical region, although the coarse grained model gives slightly better agreement with experimental data for both choices of $q_{c}$ used in this 
work. Recently, $\frac{29}{}$ another optimized version of the EPM2 model has been proposed in which the atomistic energies, lengths and charges have been rescaled to optimize agreement with the coexistence experiments. As a consequence, the agreement with experimental results is very good, in particular for the coexistence pressure (see $\diamond$ in Fig. 7). Simulations fit the experimental curve perfectly below $270 \mathrm{~K}$, while for higher temperature small deviations appear. In Ref. 33, two center Lennard-Jones models which include a quadrupolar point have been studied extensively, and coexistence densities and pressure were obtained. Tuning atomistic parameters, the agreement with the experimental curve has been optimized ${ }^{34}$ without any physical input. As a result, a quadrupolar moment for $\mathrm{CO}_{2}$ predicted in Ref. 34 equals $|Q|=3.7938 \mathrm{D} \AA$ which is quite off from the experimental value $4.3 \mathrm{D} \AA$. Finally, there is also a recent simulation, $\underline{30}$ which uses two ab-initio potentials named BBV $\underline{31}$ (denoted by $\square$ in Figs. 6 and (7) and SAPT-s² (denoted by $\circ$ in Figs. 6] and 7). Results are quite off the respective experimental values, but unlike to the previously mentioned models, no fitting procedures have been applied. No data on the interfacial free energy of the atomistic model are available so far to which we could compare our results. Figs. 6 and 7 demonstrate that the rescaled atomistic model agrees better with experiment than the simple LJ model which ignores the quadrupolar interaction completely. ${ }^{8} \underline{10}$ However, in comparison with the present model (Eq. (5) ), the atomistic models offer no advantages, even if one rescales the parameters to match the critical point. In fact, the use of Coulomb interactions in the atomistic models makes the code considerably slower.

\section{OTHER QUADRUPOLAR FLUIDS}

For a detailed discussion on how to derive simulation parameters for an arbitrary quadrupolar substance, the reader is referred to appendix B. Here we would like to focus on testing the model for other quadrupolar substances. Using literature data for $Q$, $T_{c, \exp }$ and $\rho_{c, \exp }$ for various molecular fluids, we can use our master curves (Fig. 1) to predict the value of $q_{c}$ and describe these fluids with our model, Eq. (15). Inserting Eq. (8) into Eq. 
(6) we obtain

$$
\begin{aligned}
q_{c} & =\frac{Q^{4}}{\left(k_{\mathrm{B}} T_{\mathrm{c}, \exp }\right)^{2}}\left[\frac{\rho_{\mathrm{c}, \exp } N_{\mathrm{A}}}{M_{\mathrm{Mol}}}\right]^{10 / 3} \frac{T_{c}^{*}\left(q_{c}\right)}{\rho_{c}^{*}\left(q_{c}\right)^{10 / 3}} \\
& \equiv \lambda_{\exp } \frac{T_{c}^{*}\left(q_{c}\right)}{\rho_{c}^{*}\left(q_{c}\right)^{10 / 3}} .
\end{aligned}
$$

Note that $\lambda_{\exp }$ contains all the experimental parameters which are required to define the model. Fig. 9 plots $q_{c}$ as a function of $\lambda_{\exp }$ for $\mathrm{CS}_{2}, \mathrm{~N}_{2}, \mathrm{CO}_{2}, \mathrm{C}_{2} \mathrm{H}_{2}$, and $\mathrm{C}_{6} \mathrm{H}_{6}$.

One recognizes immediately that for $\mathrm{N}_{2}$ and $\mathrm{CS}_{2}$ the effects of the quadrupolar interactions can only be minor, since $q_{c}$ is very small. Consequently, the simple LJ model (where quadrupolar effects are completely neglected) should be a reasonable description of the coexistence densities, coexistence pressures, and interfacial free energies of those fluids. Fixing the LJ parameters for $\mathrm{N}_{2}$ via $T_{c}$ and $\rho_{c}$ as done in our previous work,,$\frac{8,10}{}$ we can test immediately this hypothesis (Fig. 10). As expected, the deviations from the simple LJ fluid are indeed much less pronounced than for $\mathrm{CO}_{2}$. Note that these deviations between the measured and the predicted coexistence curves for these fluids with small $q_{c}$ are comparable to the deviations found between the simple Lennard-Jones coexistence curve and the experimental results for noble gases such as $\mathrm{Ne}$, Ar, $\mathrm{Kr}$ and Xe. These systems are considered to be the best experimental realization of a Lennard-Jones fluid (Fig. 11). In a rescaled representation $\left(T / T_{c}\right.$ plotted vs. $\left.\rho / \rho_{c}\right)$, however, the various noble gases do not exactly satisfy a "law of corresponding states". This implies that even for systems with perfectly spherical atoms, a description in terms of (classical) point particles interacting with purely pairwise potentials of the same functional form (with one parameter for the strength and another for the range) is not strictly valid.

These small deviations may be due to the need for three-body forces $\frac{67}{}$, or quantum corrections which account for differences in atomic masses. The inclusion of the three body interaction is computationally extremely expensive. Indeed in the evaluation of the total energy of the system one would need to evaluate a total number of contributions that scales like $\mathrm{N}^{3}$ instead of $\mathrm{N}^{2}$ as for the two body interactions ( $\mathrm{N}$, being the total number of molecules). For this reason the inclusion of such effects in our simple (and cheap) modeling is out of discussion, especially in view of more complicated polymer solution applications. There are several attempts $\frac{68,69}{6 h i c h}$ try to capture the three body interaction in an effective (density dependent) two body interaction. These methods cannot be used in non homogeneous fluids 
and generally where strong density fluctuations are present, like near the critical point. The fact that the method proposed in this work is based on a careful investigation of the critical points of the coarse grained models invalidates the scheme proposed in Ref. 68,69. However in Ref. 67 a quantitative estimate of the effects of the three body interaction is given starting from a careful scaling investigation of the rectilinear diameter (14)

$$
\frac{\rho_{d}(T)}{\rho_{d}\left(T_{c}\right)}=1+A_{1-\alpha}\left(1-\frac{T}{T_{c}}\right)^{(1-\alpha)}+A_{1}\left(1-\frac{T}{T_{c}}\right)+\cdots
$$

with $\alpha \approx 0.11$. The authors shows that in Eq. (18) $A_{1-\alpha}$ is related to the field mixing effect (indeed the lack of the particle hole symmetry), while $A_{1}$ could give an estimate of the three body interaction. A Mean Field van der Waals equation predicts 67 $A_{1}=2 / 5$. Deviations of the experimental data from this law of corresponding states $\left(A_{1}=2 / 5\right)$ are supposed to be related to the emergence of another energy scale like that of three body interactions. Fig. 4 of Ref. 67 suggests (for $\left.\mathrm{CO}_{2}\right) A_{1} \approx 0.95$ which differs significantly from the van der Waals value $A_{1}=0.4$ but is comparable with other fluids in particular Xenon. Comparing now the predictions for Xenon (Fig. 11) and Carbon Dioxide (Fig. 31), one can easily conclude that in our case the quadrupolar interactions are much more relevant than three body interactions.

For the sake of completeness, in Fig. 11 we have also included the full LJ potential. In an unscaled representation one would of course observe large differences between the results for the full Lennard-Jones potential and those for the cut-and-shift Lennard-Jones potential. In a scaled representation these differences vanish almost completely (except for small densities on the gas branch of the binodal) so that due to its computational efficiency the cut-and-shift potential should be preferred in coarse-grained simulations.

The case of benzene $\left(\mathrm{C}_{6} \mathrm{H}_{6}\right)$ is even more interesting. Depending on which experimental value is adopted for $Q$, one finds $q_{c}$ in the range from $q_{c}=0.121$ (for $Q=10 \mathrm{D} \AA$ ) to $q_{c}=0.247$ (for $Q=12 \mathrm{D} \AA$ ). Fig. 12 compares experimental values for the coexistence densities, coexistence pressure and interfacial tension with our predictions, using $q_{c}=0.247$. In this case we also observe a clear improvement of the agreement with experimental data with respect to the pure Lennard Jones case $\left(q_{c}=0\right.$ in Fig. 12). Deviations are of the same order of magnitude as for nitrogen (Fig. 10) and noble gases (Fig. 11). 


\section{PREDICTIONS FOR THE EQUATION OF STATE RESULTING FROM PER- TURBATION THEORY (PT)}

In this section we present results for coexistence densities and coexistence pressures (Fig. 13), which were obtained analytically using an equation of state ${ }^{70}$ in the Mean Spherical Approximation (PT-MSA) ${ }^{70}$. As is well-known ${ }^{71}$ such approaches should work well at temperatures and densities away from the critical region $\underline{\underline{71}}$ This expectation is reconfirmed by our results (Fig. 13), which show good agreement at temperatures below $0.9 T_{c}$. For $0.9 T_{c} \leq T \leq 1.2 T_{c}$, there are distinct deviations between simulations and theory because PT-MSA overestimates the critical temperature by about $10 \%$ and furthermore the slope of the binodal in the critical region is mean-field-like in PT-MSA and Ising-like in the simulation. For low temperatures the deviations are quantitatively smaller, however, the MC results and PT-MSA results cross at $T^{*} \approx 1$ (if $q_{c}=0.387$ ) and $T^{*} \approx 1.05$ (if $q_{c}=0.470$ ) on the liquid branch. Note that our comparison involves no adjustable parameter whatsoever. For many practical applications one will be interested in the temperatures and/or densities outside the critical region. Hence, the results shown in Fig. 13 are encouraging in that a relatively simple analytic method such as PT-MSA (see Appendix A for some details on this method) works well as a description of the equation of state for molecular fluids like $\mathrm{CO}_{2}$ away from the critical region if an isotropic quadrupolar interaction is included. To some extent this minimizes the need for massive Monte Carlo (MC) efforts to explore phase space. Even though MC simulations are required to determine $\varepsilon, \sigma$ and $q_{c}$ from $T_{c, \exp }, \rho_{c, \exp }$ and Q, the results are already contained in Fig. 1 and Eqs. (9a, 9b). Therefore, no new efforts with MC simulations will be needed for any future applications of PT-MSA in the context of our model.

\section{CONCLUSIONS}

In the present work, the thermodynamic properties of a coarse-grained model for quadrupolar fluids were investigated. A particular emphasis was put on the question to which extent the equation of state and the interfacial tension between coexisting vapor and liquid phases can be described accurately.

The aim of this work hence is not a chemically detailed modeling of quadrupolar fluids 
on an atomistic level, but rather to derive a model which is bot simple and accurate enough that it can serve as a starting point for the description of binary fluid mixture, solvents in polymer solutions, etc.. Obtaining efficient models for such purposes is a topic of great current interest.

As experimental input parameters, our description only requires knowledge of the experimental critical temperature $T_{c, \exp }$ and the critical density $\rho_{c, \exp }$ of the fluid and the experimental quadrupole moment $Q$ of the molecule. The quadrupolar interaction is treated in a spherical approximation $\underline{39,40}$ which can be derived from thermodynamic perturbation theory. This leads to an effective potential proportional to $Q^{4} /\left(\operatorname{Tr}_{i j}^{10}\right)$, where $T$ denotes the temperature and $r_{i j}$ the distance between the centers of mass of molecules $i$ and $j$. The application of the isotropic quadrupolar interaction is mainly motivated by the desire to have a very fast simulation code. Steric and dispersion forces are simply modeled by a Lennard-Jones potential involving parameters $\varepsilon$ and $\sigma$, which define the strength and the range of the interaction, respectively. In practice, the potential is cut and shifted to zero at a cutoff range $r_{c}=2 \sqrt[6]{2}$, which is again motivated by our desire to speed up calculations. We also provide evidence that this particular approximation mostly affects the conversion factor from $\varepsilon$ to experimental temperature and hence does not alter results significantly.

For the description of a real system, simulation parameters $\varepsilon, \sigma$ and $q_{c}=$ $Q^{4} /\left(\varepsilon \sigma^{10} k_{B} T_{c, \exp }\right)$ need to be determined from experimental values $T_{c, \exp }, \rho_{c, \exp }$ and $Q$ in physical units. To address this problem, we have determined master curves $T_{c}^{*}\left(q_{c}\right) / T_{c}^{*}(0)$ and $\rho_{c}^{*}\left(q_{c}\right) / \rho_{c}^{*}(0)$ as a function of $q_{c}$ (Fig. 1, Eqs. (9a), (9b)). This task is performed easily using grand-canonical Monte Carlo simulations $\underline{43,44,45}$ in combination with reweighting, successive umbrella sampling ${ }^{59}$ and finite-size scaling methods $\underline{45,46,47}$. With modest computational effort, these master curves are determined with a relative accuracy which is distinctly better than $1 \%$.

Carbon dioxide is a prototype of a linear elongated molecule with a rather large quadrupole moment. Comparing our predictions for the coexistence curve, vapor pressure at coexistence and interfacial tension with corresponding experimental data ${ }^{66}$, we found encouragingly good agreement (Figs. 314,5). Note that after having fixed the scales for temperature and density via $\varepsilon$ and $\sigma$, no further parameters need to be adjusted, neither for the pressure (Fig (4), nor for the interfacial tension (Fig. [5). The level of agreement which we have achieved is clearly nontrivial. However, the inclusion of quadrupolar effects is essential 
to the model and agreement with experiments deteriorates significantly if $\mathrm{CO}_{2}$ is described by a Lennard-Jones particle without quadrupole moment.

Our model produces rather accurate off-critical isotherms, too. As expected, the comparisons also reveal small discrepancies, since such a simple model cannot be absolutely perfect. However, a more realistic model, based on an all atom description of $\mathrm{CO}_{2}$ which involves considerably more complicated potentials, performs distinctly worse in comparison to our model - except if experimental critical parameters are used to empirically re-calibrate the atomistic potential. In our view, such a procedure looses the advantage of a fully predictive modeling that does not need experimental input. Complicated atomistic models also lead to rather slow simulation programs (partial charges require to deal with rather long range coulombic interactions, etc.). While such models may still be manageable for the simulation of pure fluids, their drawbacks become clearly apparent when the approach is extended to binary or ternary fluids. In mixtures, a large control parameter space needs to be explored and several phase separations may compete with each other, leading to very involved phase diagrams.

We emphasize that our successful description of carbon dioxide is by no means accidental. As a counterpart, we also consider nitrogen, a fluid with a considerably smaller quadrupole moment. In this case, a simple Lennard-Jones model with no quadrupolar forces should provide an equally good description, and in fact it does. The deviations are comparable to the deviations found between the coexistence curve of "Lennard-Jonesium" and those of various noble gases (that do not superimpose precisely in a re-scaled representation shown in Fig. 11 either.) This indicates that a simple pair potential with two parameters for the scales of energy and range does not suffice even for these prototypes of simple spherical atoms.

As a further example, we also present a comparison between our model and experimental data for benzene $\left(\mathrm{C}_{6} \mathrm{H}_{6}\right)$. Again, the agreement is very good. This result is of great interest, since the shape of the benzene molecule differs considerably from $\mathrm{CO}_{2}$, consisting of a disk rather than an elongated ellipsoid.

A very interesting question is the extent to which this concept can actually be carried over from simple fluids to binary mixtures and polymer-solvent systems. Are interactions between different types of molecules captured by simple Lorentz-Berthelot mixing rules, when one describes the pure constituents with the quality of the present work? We shall 
address this very interesting and potentially practically useful question in a forthcoming paper. We also hope that the present work will stimulate some analytical research, starting from general statistical mechanics of fluids, to provide a better theoretical understanding for the high accuracy of our approach. We also point out that the knowledge of the appropriate parameters $\varepsilon, \sigma$ and $q_{\mathrm{c}}$ allows a rather accurate description of the equation of state by liquidstate perturbation theories at state points sufficiently away from the critical region (Sec. V).

\section{ACKNOWLEDGEMENTS}

CPU times was provided by the NIC Jülich and the ZDV Mainz. We would like to thank F.Heilmann and H.Weiss of BASF AG (Ludwigshafen) for fruitful discussions and E.Müller (London) for $\mathrm{CO}_{2}$ simulations data of IMP model. BMM would also like to acknowledge BASF AG (Ludwigshafen) for financial support and J. Horbach for useful discussions. LGM wishes to acknowledge support from Ministerio de Educacion y Ciencia (project FIS2007-66079-C02-00) and Comunidad Autonoma de Madrid (project MOSSNOHO-S0505/ESP/0299).

\section{APPENDIX A: MEAN SPHERICAL APPROXIMATION (MSA) PREDICTIONS}

In this appendix we want to give some technical details concerning the analytical predictions presented in this paper. For more details we refer to the original literature. In particular, the equation of state (EOS) used in this work is a straightforward generalization

of the EOS given in appendix B of Ref. 70 for the case in which four Yukawa tails are used instead of two. We follow the strategies of Refs. 72,73 in which the Ornstein-Zernike (OZ) equation is solved in a first order MSA closure. The general idea ${ }^{71}$ is to divide the potential into a repulsive part (that becomes the reference potential) plus a perturbative attractive part

$$
U_{\lambda}(r)=\left\{\begin{array}{l}
U_{\mathrm{rep}}(r) \text { if } r<\sigma_{0} \\
\lambda U_{\mathrm{att}}(r) \text { if } \sigma_{0}<r<r_{\mathrm{cut}},
\end{array}\right.
$$

where $U\left(\sigma_{0}\right)=0, U_{\text {rep }}(r)>0, U_{\text {att }}(r)<0$ and $\lambda$ is the perturbative parameter. The reference system $(\lambda=0)$ is modeled by hard spheres with a proper radius $d_{\mathrm{HS}}, \stackrel{74}{ }$ computed using $U_{\text {rep }}{ }^{74}$. In order to get corrections to the reference free energy $A_{\text {ref }}$, a systematic expansion in $\lambda$ is developed (the general expression for $A-A_{\text {ref }}$ is standard and can be 
found for example in Ref. 70 (Eq. B5)). The explicit solution up to second order in $\lambda$ has been obtained in. Refs. 72,73 The key point developed in Ref. 73 is to fit $U_{\text {att }}$ with a couple of Yukawa tails. In the case of the LJ potential this yields

$$
\begin{aligned}
U_{\mathrm{att}}^{\mathrm{LJ}} & \approx-c_{1} \frac{e^{-z_{1}\left(r-\sigma_{0}\right)}}{r}+c_{2} \frac{e^{-z_{2}\left(r-\sigma_{0}\right)}}{r} \\
& \equiv \mathcal{Y}^{\mathrm{LJ}}\left(c_{i}, z_{i}, \sigma_{0} ; r\right) .
\end{aligned}
$$

In this work the LJ part of the potential is fitted using the same Yukawa tail as reported in Ref. 70 (Eq. B6). Equation (A2) allows us to invert some Laplace transforms that are present in the Tang-Lu solution ${ }^{72}$ and to obtain an analytical expression for the free energy which is explicitly given in Eq. B7-B10 of Ref. 70 for the apolar-fluid case $q=0$.

For the general case $q \neq 0$, Eq. (A1) will induce the same decomposition on both the LJ part and quadrupolar part of the potential

$$
U_{\mathrm{att}(\mathrm{rep})}=U_{\mathrm{att}(\mathrm{rep})}^{\mathrm{LJ}}-\frac{7}{20} q U_{\mathrm{att}(\mathrm{rep})}^{\mathrm{IQQ}} .
$$

In (A3) we have used two more Yukawa tails to fit the quadrupolar interaction $U_{\text {att }}^{\mathrm{IQQ}}$

$$
\begin{aligned}
U_{\mathrm{att}}^{\mathrm{IQQ}} & \approx-c_{3} \frac{e^{-z_{3}\left(r-\sigma_{0}\right)}}{r}+c_{4} \frac{e^{-z_{4}\left(r-\sigma_{0}\right)}}{r} \\
& \equiv \mathcal{Y}^{\mathrm{QQ}}\left(c_{i}, z_{i}, \sigma_{0} ; r\right) .
\end{aligned}
$$

Because $q\left(=q_{c} T_{c} / T\right)$ is factored out in (A3), $c_{3,4}$ and $z_{3,4}$ do not depend on temperature $T$. This is an important simplification because using (A2) and (A4) we can get an immediate fit for $U_{\text {att }}(\mathrm{A} 3)$ for every $q$ and $T$

$$
U_{\mathrm{att}} \approx \mathcal{Y}^{\mathrm{LJ}}\left(c_{i}, z_{i}, \sigma_{0} ; r\right)-\frac{7}{20} q \mathcal{Y}^{\mathrm{QQ}}\left(c_{i}, z_{i}, \sigma_{0} ; r\right)
$$

By using the previous fit (A5) and extending Eq. B7-B10 in Ref. 70 to the case in which more than two Yukawa expressions are used to fit the potential, we have obtained the desired EOS used in the present work.

\section{APPENDIX B: DETERMINATION OF SIMULATION PARAMETERS}

Simulation parameters $\varepsilon, \sigma$ and $\mathrm{q}_{c}$ are needed to convert simulation units into experimental units. Knowledge of $\mathrm{q}_{c}$, or rather $q=q_{c} \cdot T_{c} / T$ is also required as input before a simulation can be started. In Table I, we have collected the simulation parameters for the 
quadrupolar substances mentioned in the paper. However, we would also like to convey some hands-on knowledge on how to calculate these parameters and extend the model to substances not listed in Table I. Furthermore, we provide fitting curves (Table III) which allow us to determine the phase diagram of an arbitrary substance without additional MC simulations.

For $\mathrm{q}_{c}=0, \varepsilon$ and $\sigma$ can be determined directly from the critical temperature $\mathrm{T}_{c}$ and the critical density $\rho_{c}$ using Eq. (8) . For $q_{c} \neq 0$, the location of the critical point itself depends on $q_{c}$. Therefore, $\varepsilon$ and $\sigma$ also depend on $q_{c}$ (Eq. (8) ), and a simple iteration procedure can be formulated. Starting with $q_{c}=0, T_{c}$ and $\rho_{c}$ are computed using the master curves from Eqs. (9a) and (9b). From these results, $\varepsilon$ and $\sigma$ are determined with Eq. (8) and a new value for $q_{c}$ with Eq. (6). The iteration is repeated until $q_{c}, \varepsilon$ and $\sigma$ converge. Usually, around 5-10 iterations are sufficient to obtain simulation parameters with good accuracy without any additional simulations. In the following, we present a pseudo-code for our $\mathrm{CO}_{2}$ calculations which can be extended to any quadrupolar substance by substituting experimental values for $\mathrm{Q}=4.3 \mathrm{D} \AA, \mathrm{T}_{c}=304.1282 \mathrm{~K}$, and $\rho_{c}=10.6249 \mathrm{~mol} / \mathrm{l}$ :

\section{$\underline{\text { Initialize variables }}$}

$$
\begin{array}{ll}
\mathrm{Q}=4.3 & / * \text { D } * / \\
\mathrm{Q}=\mathrm{Q}^{*} 3.33564^{*} 10^{-40} ; & /^{*} \text { convert Q to SI units */ } \\
\mathrm{T}_{\mathrm{c}, \exp }=304.1282 ; & /^{*} \mathrm{~K} * / \\
\mathrm{rho}_{\mathrm{c}, \exp }=10.6249 ; & /^{*} \mathrm{~mol} / \mathrm{l} * / \\
\mathrm{T}_{\mathrm{LJ}}(\mathrm{q}=0)=0.99821 & / * \text { critical temperature of simulation for } \mathrm{q}=0 * / \\
\operatorname{rho}_{\mathrm{LJ}}(\mathrm{q}=0)=0.32276 & / * \text { critical density of simulation for } \mathrm{q}=0 * / \\
\mathrm{q}=0 ; &
\end{array}
$$

\section{Iteration}

$$
\begin{aligned}
& \text { for }(\mathrm{i}=0 ; \mathrm{i}<20 ; \mathrm{i}++)\{ \\
& \mathrm{T}=\mathrm{T}_{\mathrm{LJ}}(\mathrm{q}=0) *\left(1+0.46111 * \mathrm{q}+0.17571 * \mathrm{q}^{2}\right) ; \quad / * \text { Eq.(9a) } * / \\
& \text { density }=\operatorname{rho}_{\mathrm{LJ}}(\mathrm{q}=0) *(1+0.19298 * \mathrm{q}) ; \quad \quad / * \text { Eq.(9b) } * / \\
& \text { epsilon }=\mathrm{T}_{\mathrm{c}, \exp } * 1.38065 * 10^{-23} / \mathrm{T} ; \quad / * \text { Eq. (88) } * / \\
& \operatorname{sigma}=\left(\mathrm{rho}_{\mathrm{c}, \exp } * 1000 * 6.02214 * 10^{23} / \text { density }\right)^{-1 / 3} ; \quad / * \text { Eq. (88) } * / \\
& \mathrm{Q}_{1}=\mathrm{Q} /\left(\operatorname{sqrt}\left(\operatorname{epsilon}^{*} \operatorname{sigma}{ }^{5}\right)\right) ; \quad \quad / * \text { Eq. (66) } * /
\end{aligned}
$$


$\mathrm{q}=\mathrm{Q}_{1}^{4} /\left(T * 1.237990147 * 10^{-20}\right) ; \quad / * \quad \mathrm{~T}_{\mathrm{sim}}=\mathrm{k}_{\mathrm{B}} \mathrm{T}_{\exp } / \varepsilon, \quad\left(4 \pi \varepsilon_{0}\right)^{2}-$ SI units $* /$

print T, epsilon, sigma, q;

\}

Alternatively, $\mathrm{q}_{c}$ can also be determined from the fitting curve in Fig. 10, $\lambda_{\exp }$ is a dimensionless parameter, which already contains all the experimental information required to define the model. If all constants are included, $\lambda_{\exp }$ reduces to

$$
\lambda_{\exp }=96.754 \cdot 10^{-5} \frac{Q^{4}}{T_{\mathrm{c}, \exp }^{2}}\left(\rho_{\mathrm{c}, \exp }\right)^{\frac{10}{3}} .
$$

In this equation, one simply needs to plug in experimental values for quadrupolar moment $Q$ in $\mathrm{D} \AA$, critical temperature $\mathrm{T}_{\mathrm{c}, \exp }$ in $\mathrm{K}$, and critical molar density $\rho_{\mathrm{c}, \exp }$ in $\mathrm{mol} / \mathrm{cm}^{3}$. $\mathrm{q}_{c}$ can be read off from Fig. 10 or determined via the following fit to the curve:

$$
q_{c}=\lambda_{\exp }\left(43.1018-266.251 \lambda_{\exp }+5047.01 \lambda_{\exp }^{2}\right) \quad \lambda_{\exp } \leq 0.02
$$

$\mathrm{T}_{c}, \rho_{c}, \varepsilon$ and $\sigma$ follow from Eqs. (9a) $(9 \mathrm{~b})$, and (8)

Finally, we demonstrate how our accumulated simulation data can be used to provide a rough estimate of the phase diagram for an arbitrary quadrupolar substance without any additional MC simulations. We simulated several values for $q_{c}$ in the range of $0.1 \leq q_{c} \leq 0.47$. Four temperatures were considered such that $T_{i}^{*}\left(q_{c}\right) / T_{c}^{*}\left(q_{c}\right)(i=1, \cdots, 4)$ is independent of $q_{c}: T_{1}^{*}=0.974499 \cdot T_{c}^{*}, T_{2}^{*}=0.932125 \cdot T_{c}^{*}, T_{3}^{*}=0.864337 \cdot T_{c}^{*}$, and $T_{4}^{*}=0.813494 \cdot T_{c}^{*}$. As indicated before, critical quantities scale almost linearly with $\mathrm{q}_{c}$ (Fig,1, Eqs.(9a) and (9b) ). During our investigations, we observed that this approximation also holds away from criticality. The corresponding fitting curves are listed in Table II.

First, one needs to determine $\varepsilon, \sigma$ and $\mathrm{q}_{c}$ for the substance in question as demonstrated in the previous section. Vapor and liquid coexistence densities, interface tension and pressure at the selected temperature can be computed by inserting $\mathrm{q}_{c}$ into the respective fitting curves. The following equations can be used to convert the results from simulation units to experimental units:

$$
T_{\text {exp }}=\frac{\varepsilon\left(q_{c}\right)}{k_{B}} T_{i}^{*}, \quad \rho_{\text {exp }, \mathrm{l}, \mathrm{g}}=\rho_{l, g}^{*} \frac{M_{\mathrm{mol}}}{N_{A} \sigma\left(q_{c}\right)^{3}}, \quad \gamma_{\exp }=\gamma^{*} \frac{\varepsilon\left(q_{c}\right)}{\sigma\left(q_{c}\right)^{2}}, \quad p_{\exp }=p^{*} \frac{\varepsilon\left(q_{c}\right)}{\sigma\left(q_{c}\right)^{3}} .
$$


* Electronic address: kurt.binder@uni-mainz.de

1 E. Kiran and Brennecke (eds.) Supercritical Fluid Engineering Science. ACS Symposium Series 514 (American Chem. Soc., Washington, D.C. 1993)

2 E. Kiran and J. M. H. Levelt-Sengers (eds.) Supercritical Fluids (Kluwer, Dordrecht, 1994)

3 M. F. Kemmere and Th. Meyer (eds.) Supercritical Carbon Dioxide in Polymer Reaction Engineering (Wiley-VCH, Weinheim 2005)

4 J. S. Rowlinson and F. L. Swinton, Liquids and Liquid Mixtures (Butterworths, London, 1982)

5 P. van Konynenburg and R. L. Scott, Philos. Trans. Soc. London Series A298, 495 (1980)

6 G. Schneider, Z. Alwani, W. Heim, E. Horvath, and E. U. Franck, Chem. Ing. Techn. 39, 649 (1967)

7 C. T. Amon, R. J. Martin, and R. Kobayashi, Fluid Phase Equilib. 31, 89 (1986)

8 P. Virnau, M. Müller, L. G. MacDowell and K. Binder, J. Chem. Phys. 121, 2169 (2004)

9 P. Virnau, M. Müller, L. G. MacDowell and K. Binder, Comp. Phys. Comm. 147, 378 (2002)

10 K. Binder, M. Müller, P. Virnau, and L. G. MacDowell, Adv. Polym. Sci. 173, 1 (2005)

11 Y. Iwai, Y. Koga, Y. Hata, H. Uchida and Y. Arai, Fluid Phase Equilib. 104, 403 (1995)

12 W. Paul, K. Binder, K. Kremer, and D. W. Heermann, Macromolecules 24, 6332 (1991)

13 W. Tschöp, K. Kremer, J. Batoulis, T. Bürger, and O. Hahn, Acta Polym. 49, 61 (1998)

14 J. Baschnagel, K. Binder, P. Doruker, A. A. Gusev, O. Hahn, K. Kremer, W. L. Mattice, F. Müller-Plathe, M. Murat, W. Paul, S. Santos, U. W. Suter, and V. Tries, Adv. Polym. Sci. 152, 41 (2000)

15 K. Kremer and F. Müller-Plathe, MRS Bulletin 26, 205 (2001)

16 F. Müller-Plathe, Chem. Phys. Chem. 3, 754 (2002)

17 C. F. Abrams and K. Kremer, Macromolecules 36, 260 (2003)

18 K. Binder, W. Paul, S. Santos and U. W. Suter, in Simlation Methods for Polymers (M. Kotelyanski and D. N. Theodorou, eds.) pp. 491-510 (M. Dekker, New York 2004)

19 G. Voth (Ed.) Coarse-Graining of Condensed Phase and Biomolecular Systems (Taylor and Francis, in press)

20 C. S. Murthy, K. Singer and I. R. McDonald, Mol. Phys. 44, 135 (1981)

21 H. J. Böhm, C. Meissner and R. Ahlrichs, Mol. Phys. 53, 651 (1984) 
22 H. J. Böhm and R. Ahlrichs, Mol. Phys. 55, 445 (1985)

23 S. B. Zhu and G. W. Robinson, Comp. Phys. Commun. 52, 317 (1989)

24 R. D. Etters and B. Kuchta, J. Phys. Chem. 90, 4537 (1989)

25 L. C. Geiger, B. M. Ladanyi and M. E. Chapin, J. Chem. Phys. 93, 4533 (1990)

26 B. J. Palmer and B. C. Garrett, J. Chem. Phys. 98, 4047 (1993)

27 J. G. Harris and K. H. Yung, J. Phys. Chem. 99, 12021 (1995)

28 J. Vorholz, V. I. Harismiadis, B. Rumpf, A. Z. Panagiotopoulos and G. Maurer, Fluid Phase Equilib. 170, 203 (2000)

29 Z. Zhang and Z. Duan, J. Chem. Phys. 122, 214507 (2005)

30 C. Bratschi, H. Huber and D. J. Searles, J. Chem. Phys. 126, 164105 (2007)

31 S. Bock, E. Bich and E. Vogel, Chem. Phys. 257, 147 (2000)

32 R. Bukowsky, J. Sadlej, B. Jeziorski, P. Jankowski, K. Szalewicz, S. A. Kucharski, H. L. Williams and B. M. Rice, J. Chem. Phys. 110, 3785 (1999)

33 J. Stoll, J. Vrabec, H. Hasse and J. Fischer, Fluid Phase Equilib. 179, 339 (2001)

34 J. Vrabec, J. Stoll and H. Hasse, J. Phys. Chem. B 105, 12126 (2001)

35 B. Garzon, S. Lago, C. Vega, E. de Miguel and L. F. Rull, J. Chem. Phys. 101, 4166 (1994)

36 CRC Handbook of Chemistry and Physics (1985) CRC Press, Boca Raton

37 A. Galindo and F. J. Blas, J. Phys. Chem. B106, 4503 (2002)

38 I. Polishuk, J. Wisniak, and H. Segura, J. Phys. Chem. B107, 1864 (2003)

39 G. Stell, J. C. Rasaiah and H. Narang, Mol. Phys. 27, 1393 (1974)

40 E. A. Müller and L. D. Gelb, Ind. Eng. Chem. Res. 42, 4123 (2003)

41 L. D. Gelb and E. A. Müller, Fluid Phase Equilib. 203, 1 (2002)

42 S. Albo and E. A. Müller, J. Phys. Chem. B107, 1672 (2003)

43 K. Binder, Rep. Progr. Phys. 60, 487 (1997)

44 D. P. Landau and K. Binder, A Guide to Monte Carlo Simulations in Statistical Physics, $2^{\text {nd }}$ ed. (Cambridge University Press, Cambridge, 2005)

45 N. B. Wilding, J. Phys. : Condensed Matter 9, 585 (1997)

46 M. E. Fisher, in Critical Phenomena, ed. M. S. Green (Academic Press, London, 1971) p.1

47 K. Binder, in Computational Methods in Field Theory, eds. C. B. Lang and H. Gausterer (Springer, Berlin, 1992)

48 A. Z. Panagiotopoulos, Mol. Sim. 9, 1 (1992) 
49 A. Z. Panagiotopoulos, Mol. Phys. 61, 813 (1987)

50 C. G. Gray and K. E. Gubbins, Theory of Molecular Fluids, Vol. I: Fundamentals. Clarendon Press, Oxford (1984)

51 D. Frenkel and B. Smit, Understanding Molecular Simulation: From Algorithms to Applications. $2^{\text {nd }}$ Ed. (Academic Press, San Diego, 2002)

52 A. M. Ferrenberg and R. H. Swendsen, Phys. Rev. Lett. 61, 2635 (1988)

53 K. Binder and D. P. Landau, Phys. Rev. B30, 1477 (1984)

54 C. Borgs and R. Kotecky, J. Stat. Phys. 60, 79 (1990)

55 Y. C. Kim, M. E. Fisher, and E. Luijten, Phys. Rev. Lett. 91, 065701 (2003); Y. C. Kim and M. E. Fisher, Computer Phys. Commun. 169, 295 (2005)

56 K. Binder, Phys. Rev. A25, 1699 (1982)

57 B. A. Berg and T. Neuhaus, Phys. Rev. Lett. 68, 9 (1992)

58 F. Wang and D. P. Landau, Phys. Rev. E64, 056101 (2001)

59 P. Virnau and M. Müller, J. Chem. Phys. 120, 10925 (2004)

60 B. A. Berg, U. Hansmann and T. Neuhaus, Phys. Rev. B47, 497 (1993)

61 M. Müller, K. Binder, and W. Oed, J. Chem. Soc. Faraday Trans. 28, 8639 (1995)

62 J. E. Hunter III and W. P. Reinhardt, J. Chem. Phys. 103, 8627 (1995).

63 J. Potoff and A. Panagiotopoulus, J. Chem. Phys. 112, 6411 (2000).

64 R. L. C. Vink, J. Horbach and K. Binder, Phys. Rev. E71, 011401 (2005); R. L. C. Vink and J. Horbach, J. Phys. : Condens. Matter 16, 3807 (2004).

65 J. Zinn-Justin, Quantum Field Theory and Critical Phenomena, $3^{\text {nd }}$ ed. (Clarendon Press, Oxford 1996).

66 NIST website: http://webbook.nist.gov/chemistry/

67 M. W. Pestak, R. E. Goldstein, M. H. W. Chan, J. R. de Bruyn and N. W. Ashcroft, Phys. Rev. B 36, 599 (1987).

68 G. Raabe and R. J. Sadus, J. Chem. Phys. 119, 6691 (2003).

69 L. Wang and R. J. Sadus, J. Chem. Phys. 125, 144509 (2006).

70 L. G. MacDowell, M. Müller, C. Vega and K. Binder, J. Chem. Phys. 113, 419 (2000).

71 J. P. Hansen, I. R. McDonald, Theory of Simple Liquids (Academic, New York, 1986).

72 Y. Tang and B. C. -Y. Lu, J. Chem. Phys. 99, 9828 (1993).

73 Y. Tang, Z. Tong and B. C.-Y. Lu, Fluid Phase Equilib. 134, 21 (1997). 
74 J. A. Barker and D. Henderson, J. Chem. Phys. 47, 4714 (1967). 
TABLE I: Experimental data and simulation parameters for several quadrupolar substances as obtained in the present work

TABLE II: Fitting curves to determine coexistence properties for an arbitrary quadrupolar substance at selected temperatures $T_{i}^{*}\left(q_{c}\right) / T_{c}^{*}\left(q_{c}\right)(i=1, \cdots, 4): T_{1}^{*}=0.974499 \cdot T_{c}^{*}, T_{2}^{*}=0.932125 \cdot T_{c}^{*}$, $T_{3}^{*}=0.864337 \cdot T_{c}^{*}$, and $T_{4}^{*}=0.813494 \cdot T_{c}^{*}($ see text $)$

FIG. 1: Master curves: normalized critical temperature $T_{c}^{*}\left(q_{c}\right) / T_{c}^{*}(0)$, normalized critical density $\rho_{c}^{*}\left(q_{c}\right) / \rho_{c}^{*}(0)$, and normalized critical pressure $p_{c}^{*}\left(q_{c}\right) / p_{c}^{*}(0)$ plotted versus the quadrupolar parameter $q_{c}$. Symbols represent simulation data, curves are the interpolating functions (Eqs. (9a) and $(\underline{9 \mathrm{~b}})$ ) and $p_{c}^{*}\left(q_{c}\right) / p_{c}^{*}(0)=\left(1+0.67423 q_{c}+0.274349 q_{c}^{2}\right)$ with $p_{c}^{*}(0)=0.087221$.

FIG. 2: Second and fourth order cumulants $U_{2}, U_{4}$ plotted for $q=0.3$ versus $T^{*}=k_{B} T / \varepsilon$ for three choices of $L$. Broken horizontal values indicate the theoretical values established for the Ising universality class $\stackrel{44,45}{ }$ From the intersections one can conclude $T_{c}^{*}=1.152 \pm 0.003$ for this particular case. Inset: the slope of the fourth order cumulants $\left(Y_{1}\right)$ as a function of the box size, on a log-log scale. The data points fall on a straight line with a slope equal to 1.584 in agreement with the finite size prediction $1 / \nu$, with $\nu \approx 0.630$ for the Ising universality class .65

FIG. 3: Coexistence curve of $\mathrm{CO}_{2}$ plotted in the temperature-density plane. The broken curve denotes the experimental data (from NIST $\underline{66}$ ), the full curve is the result for the LJ model without quadrupolar interactions ${ }^{8}$. Solid square denotes the critical point of $\mathrm{CO}_{2} \cdot(\times)$ and $(*)$ are the results of the present $\mu V T$ work for two choices of $q_{c}=q\left(T_{c}\right)$ as indicated in the figure. (०) are the results of the spherical averaged model investigated in Ref. 42.

FIG. 4: Coexistence pressure of $\mathrm{CO}_{2}$ plotted vs. temperature. The broken curve denotes the experimental data,$\frac{66}{}$ the full curve: the results for the LJ model without quadrupolar interactions. $(\times)$ and $(*)$ are the results of the present NVT work for two choices of $q_{c}=q\left(T_{c}\right)$ as indicated in the figure. 
FIG. 5: Interface tension $\gamma(T)$ of $\mathrm{CO}_{2}$ plotted vs. temperature. The broken curve denotes the experimental data (from NIST $\underline{66}$ ), the full curve: the results for the LJ model without quadrupolar interactions $\underline{\underline{8}}(\times)$ and $(*)$ are the results of the present work for two choices of $q_{c}=q\left(T_{c}\right)$ as indicated in the figure.

FIG. 6: Coexistence curve of $\mathrm{CO}_{2}$ plotted in the temperature-density plane. The broken curve denotes the experimental data (from NIST $\underline{66}$ ), the full curve: the results for LJ model without quadrupolar interactions ${ }^{8}$. $(\bullet)$ denotes the critical point of $\mathrm{CO}_{2} .(*)$ and $(\times)$ denote the results of this work for $q_{c}=0.387$ and $q_{c}=0.470$, respectively. $(+)$ are the results of the EPM model introduced in Ref. 27. $(\nabla)$ are the results from Ref. 27 for the EPM model with flexible molecules, which give essentially the same thermodynamic properties as the rigid molecules. $(\triangleleft)$ are the results of Ref. 27 for the rescaled EPM model (EPM2). (o) and ( $\square$ ) correspond to simulations 30 of two ab initio potentials. $\frac{31,32}{32}$

FIG. 7: Coexistence pressure of $\mathrm{CO}_{2}$ plotted vs. temperature. Labeling of curves and symbols is the same as in Fig. 6. We also show simulations of an optimized EPM2 model 29 (see $\diamond)$ which is in good agreement with experiments. We stress that the nice agreement of our model with experiments near the critical point is not given a priory because our method only fixes the critical temperature and the critical density.

FIG. 8: Supercritical isobar for $\mathrm{p}=200$ bar. The broken curve denotes the experimental data $\underline{66}$ $(\times)$ and $(*)$ are the results of the present NVT work for two choices of $q_{c}=q\left(T_{c}\right)$ as indicated in the figure. $(\triangleleft)$ are the prediction of the atomistic EPM2 model given in Ref. 28. The coexistence curve near the critical point is also reported.

FIG. 9: Estimates for the quadrupolar parameter $q_{c}$ for various quadrupolar fluids characterized by parameter $\lambda_{\exp }$ (Eq. (17)). The corresponding experimentally measured quadrupole moments $Q$ of these systems are quoted in brackets (see also Table \). 
FIG. 10: Lennard-Jones results $\left(q_{c}=0\right)$ for $\mathrm{N}_{2}$. From top to bottom: coexistence curve in the temperature-density plane, vapor pressure vs. temperature and interface tension vs. temperature. Symbols correspond to simulations of a simple Lennard-Jones model without quadrupolar moment obtained from $\mu \mathrm{VT}$ simulations $\frac{8}{8}$ (coexistence densities and interface tensions) and NVT simulations (pressure). The broken curves denote the experimental data (from NIST $\underline{66}$ ).

FIG. 11: Coexistence curves $\left(T / T_{c}\right.$ plotted vs. $\left.\rho / \rho_{c}\right)$ for various noble gases in comparison with the prediction of the cut-and-shifted Lennard-Jones model (LJ) $)^{\frac{8}{}}$ and the full Lennard-Jones model. $\underline{\underline{63}}$

FIG. 12: New predictions for benzene $\left(\mathrm{C}_{6} \mathrm{H}_{6}\right)$. From top to bottom: coexistence curve in the temperature-density plane, vapor pressure vs. temperature and interface tension vs. temperature. The broken curves denote the experimental data, $\frac{66}{6}$ the full curve is the result of the simple LennardJones model. $(\triangleright)$ denote the present results which include an isotropic quadrupolar interaction for $q_{c}=q\left(T_{c}\right)$ corresponding to $Q=12 \mathrm{D} \AA$.

FIG. 13: Coexistence densities and coexistence vapor pressure: a comparison between the MC simulations and the PT-MSA prediction. The two choices of $q_{c}=q\left(T_{c}\right)$ used in this work are included as indicated. 


\begin{tabular}{|c|c|c|c|c|c|c|c|}
\hline Subst. & $\mathrm{Q}[\mathrm{D} \AA]$ & $T_{c, \exp }[\mathrm{K}]$ & $\rho_{c, \exp }[\mathrm{mol} / \mathrm{l}]$ & $\lambda_{\exp }$ & $q_{c}$ & $\varepsilon / k_{\mathrm{B}}[\mathrm{K}]$ & $\sigma[\AA]$ \\
\hline $\mathrm{CO}_{2}$ & 4.3 & 304.1282 & 10.6249 & 0.009430 & 0.387 & 252.829 & 3.785 \\
\hline $\mathrm{CS}_{2}$ & 3.6 & 552 & 5.78 & 0.0001848 & 0.0080 & 550.95 & 4.528 \\
\hline $\mathrm{N}_{2}$ & 1.47 & 126.2 & 11.18 & 0.0008864 & 0.038 & 124.208 & 3.642 \\
& 0 & 126.2 & 11.18 & 0 & 0 & 126.426 & 3.633 \\
\hline $\mathrm{C}_{2} \mathrm{H}_{2}$ & 5.5 & 308.3 & 8.913 & 0.013775 & 0.553 & 235.942 & 4.052 \\
\hline $\mathrm{C}_{6} \mathrm{H}_{6}$ & 12 & 562 & 3.9 & 0.0059311 & 0.247 & 500.468 & 5.242 \\
\hline
\end{tabular}

TABLE I 


\begin{tabular}{|c|c|}
\hline Observable & Fitting Formula \\
\hline$\rho_{1, g}^{*}$ & $\approx 0.162$ \\
$\rho_{2, g}^{*}$ & $0.099506-0.0094827 q_{c}$ \\
$\rho_{3, g}^{*}$ & $0.055372-0.017106 q_{c}$ \\
$\rho_{4, g}^{*}$ & $0.036003-0.018 q_{c}$ \\
\hline$\rho_{1, l}^{*}$ & $0.49215+0.12426 q_{c}+0.021146 q_{c}^{2}$ \\
$\rho_{2, l}^{*}$ & $0.57055+0.15313 q_{c}+0.025081 q_{c}^{2}$ \\
$\rho_{3, l}^{*}$ & $0.64597+0.1531 q_{c}+0.09854 q_{c}^{2}$ \\
$\rho_{4, l}^{*}$ & $0.68355+0.22094 q_{c}+0.042765 q_{c}^{2}$ \\
\hline$\gamma_{1}^{*}$ & $0.020384+0.016672 q_{c}+0.027991 q_{c}^{2}$ \\
$\gamma_{2}^{*}$ & $0.068376+0.072064 q_{c}+0.082864 q_{c}^{2}$ \\
$\gamma_{3}^{*}$ & $0.16187+0.19493 q_{c}+0.18704 q_{c}^{2}$ \\
$\gamma_{4}^{*}$ & $0.23945+0.29931 q_{c}+0.30352 q_{c}^{2}$ \\
\hline$p_{1}^{*}$ & $0.075861+0.041526 q_{c}+0.024072 q_{c}^{2}$ \\
$p_{2}^{*}$ & $0.056804+0.026873 q_{c}+0.011408 q_{c}^{2}$ \\
$p_{3}^{*}$ & $0.035115+0.0099939 q_{c}+0.00067637 q_{c}^{2}$ \\
$p_{4}^{*}$ & $0.023617+0.0010425 q_{c}-0.0009939 q_{c}^{2}$ \\
\hline
\end{tabular}

TABLE II 


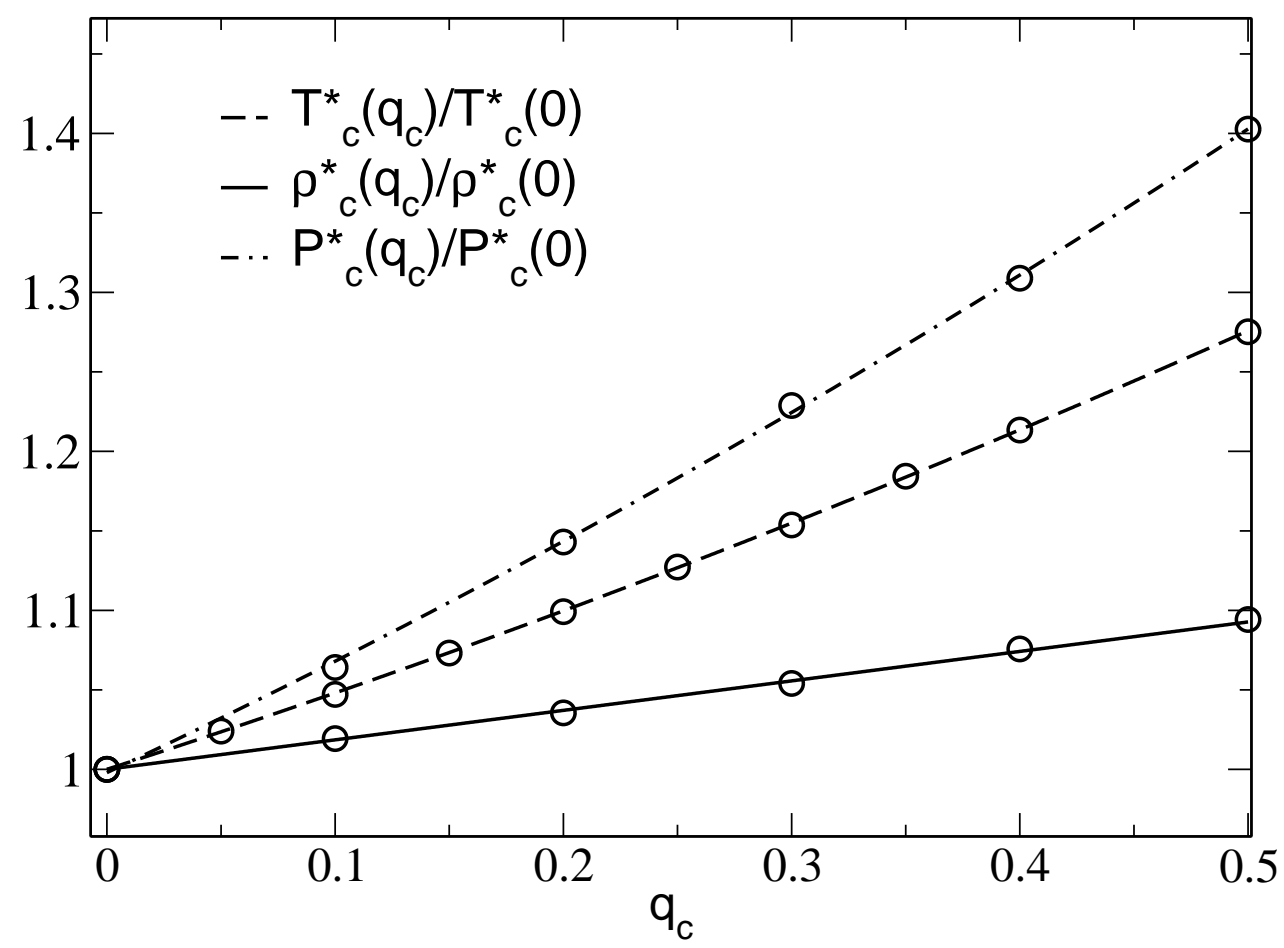

FIG. 1 


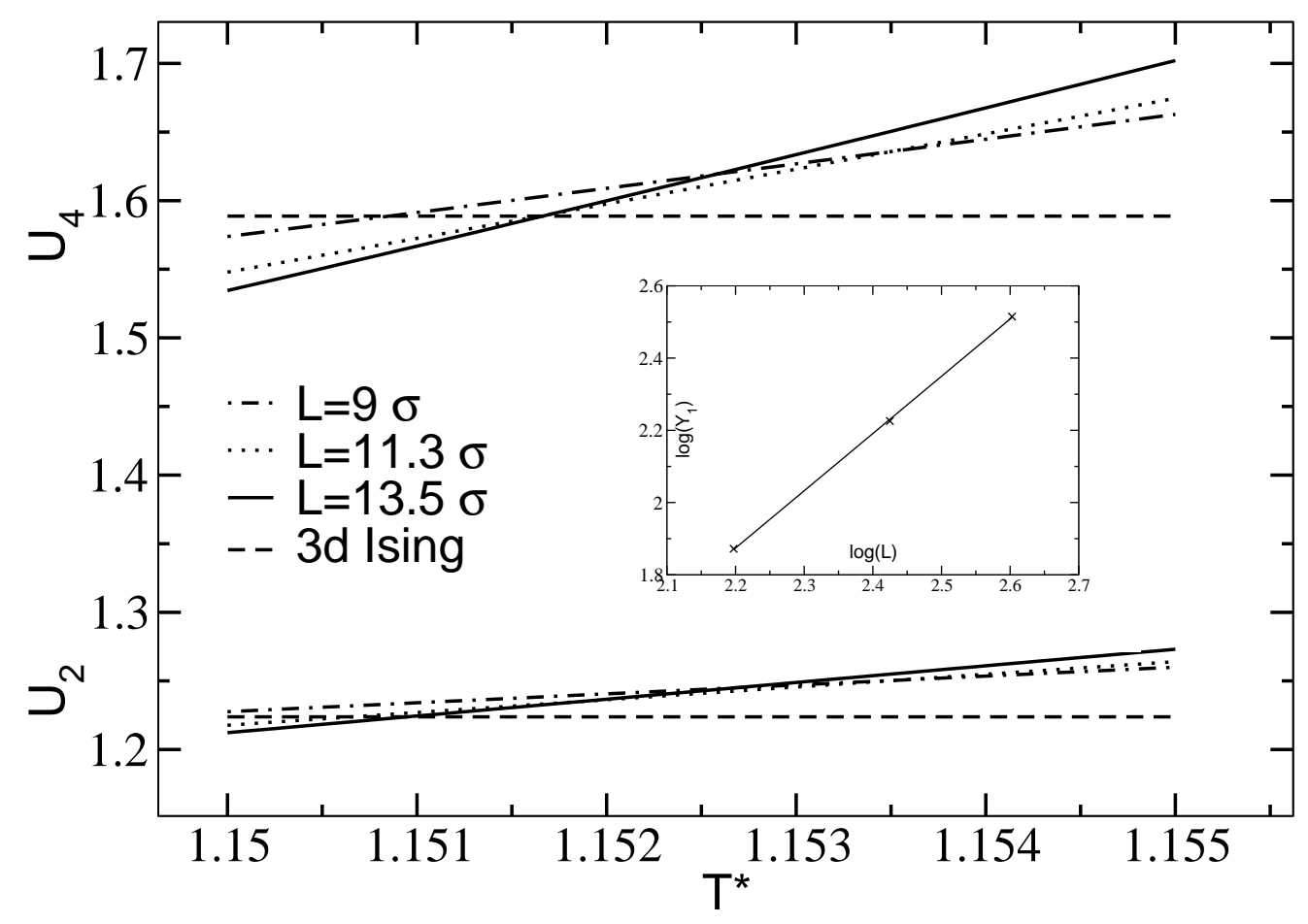

FIG. 2 


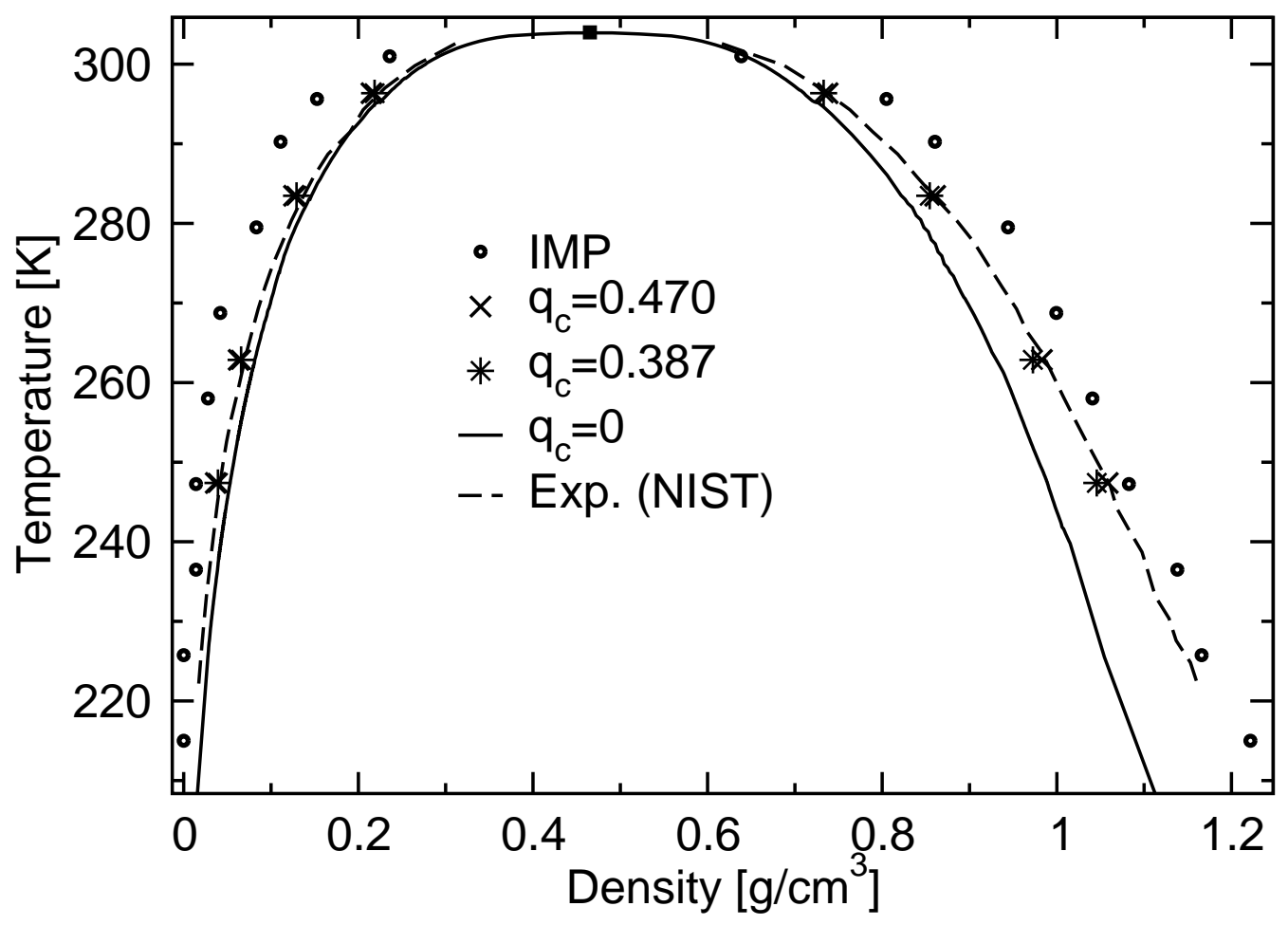

FIG. 3 


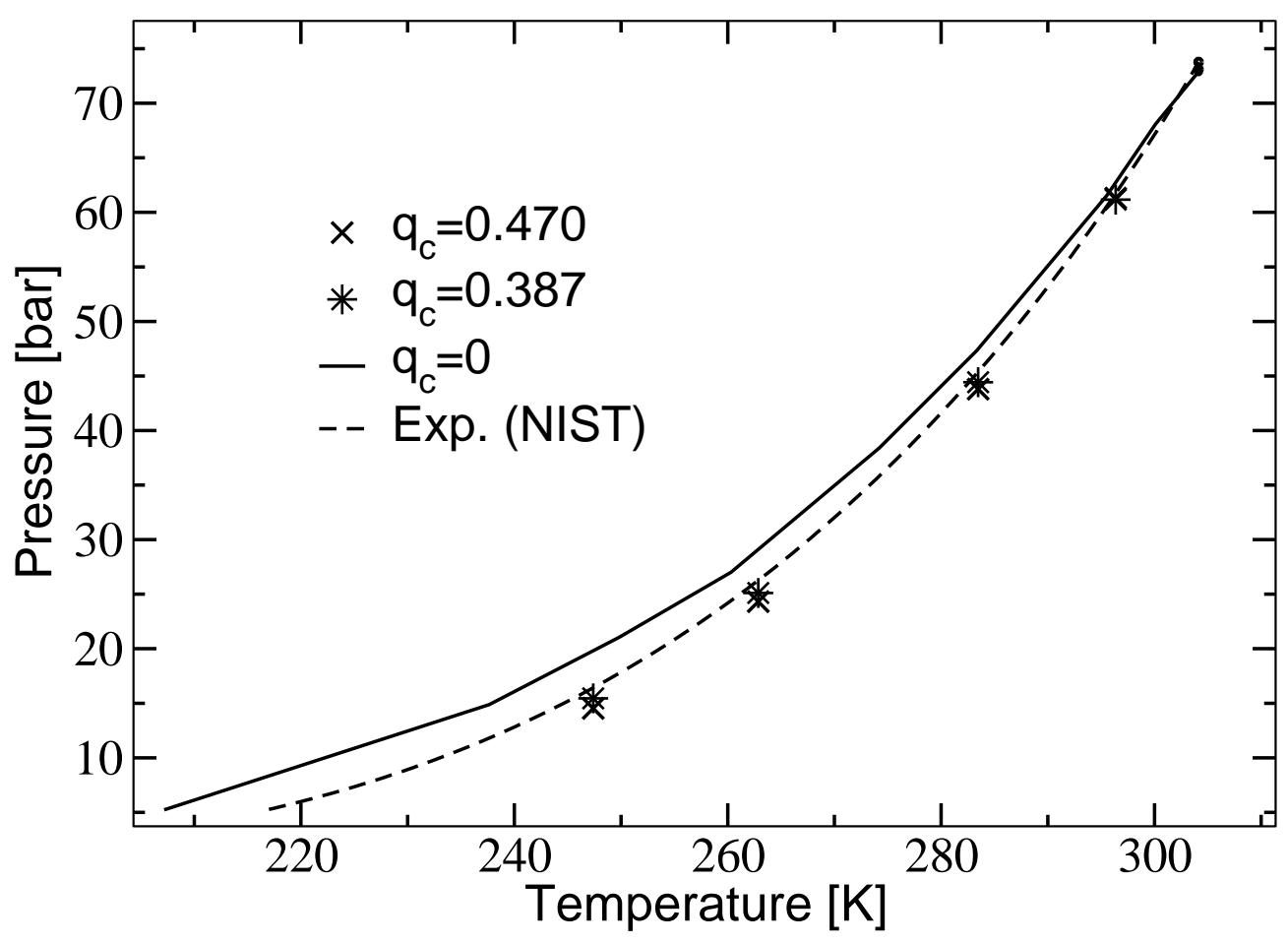

FIG. 4 


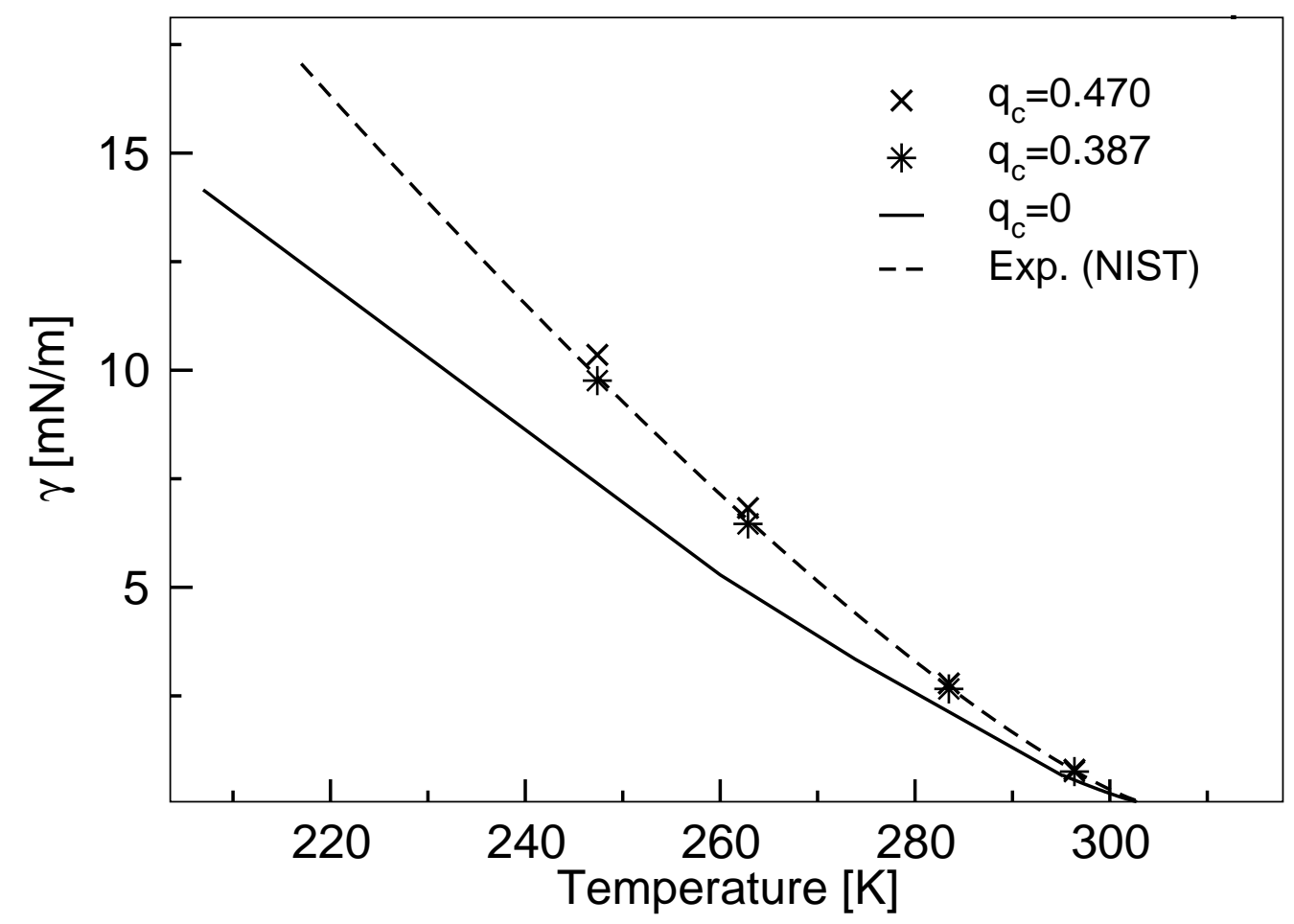

FIG. 5 


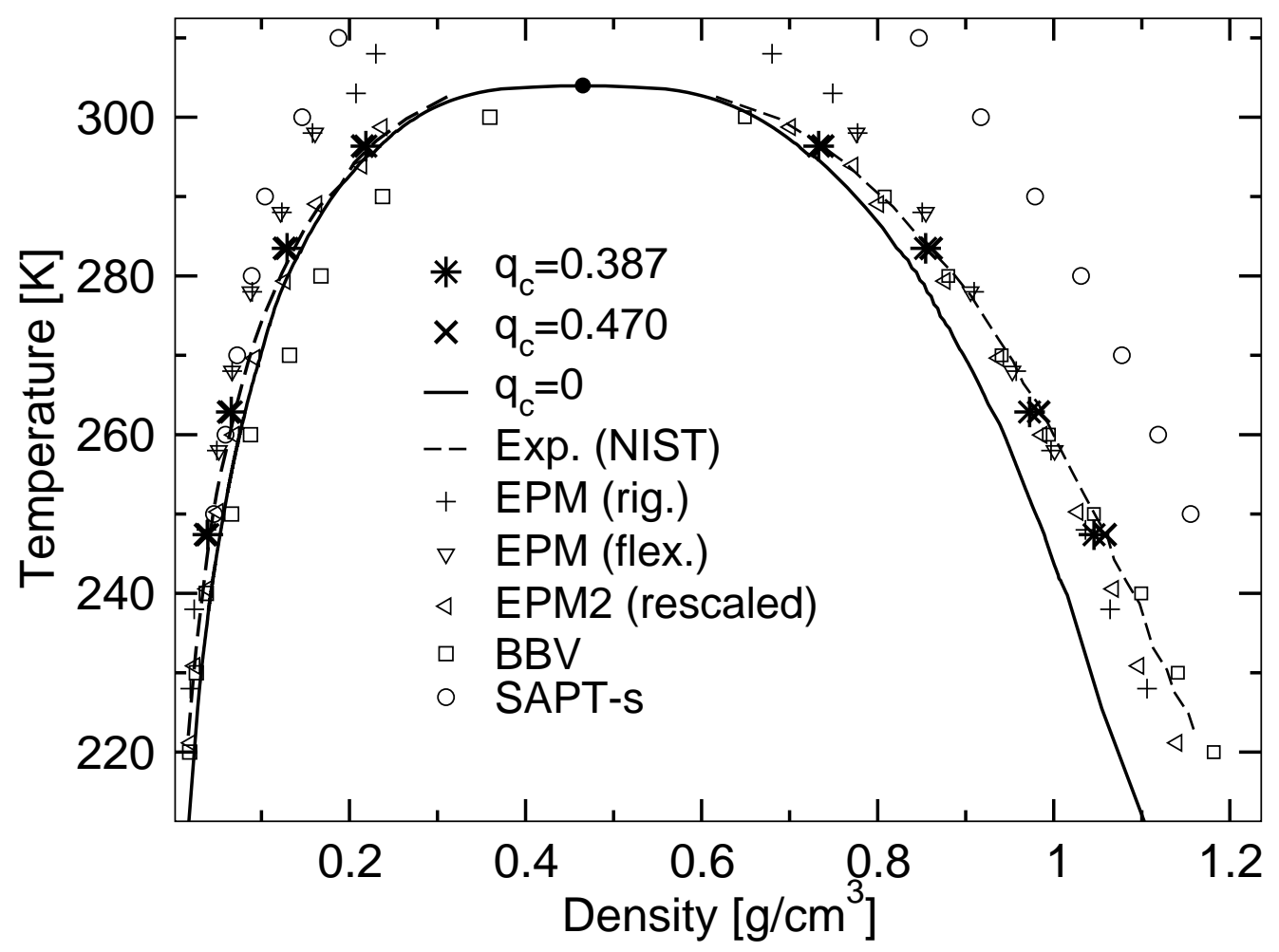

FIG. 6 


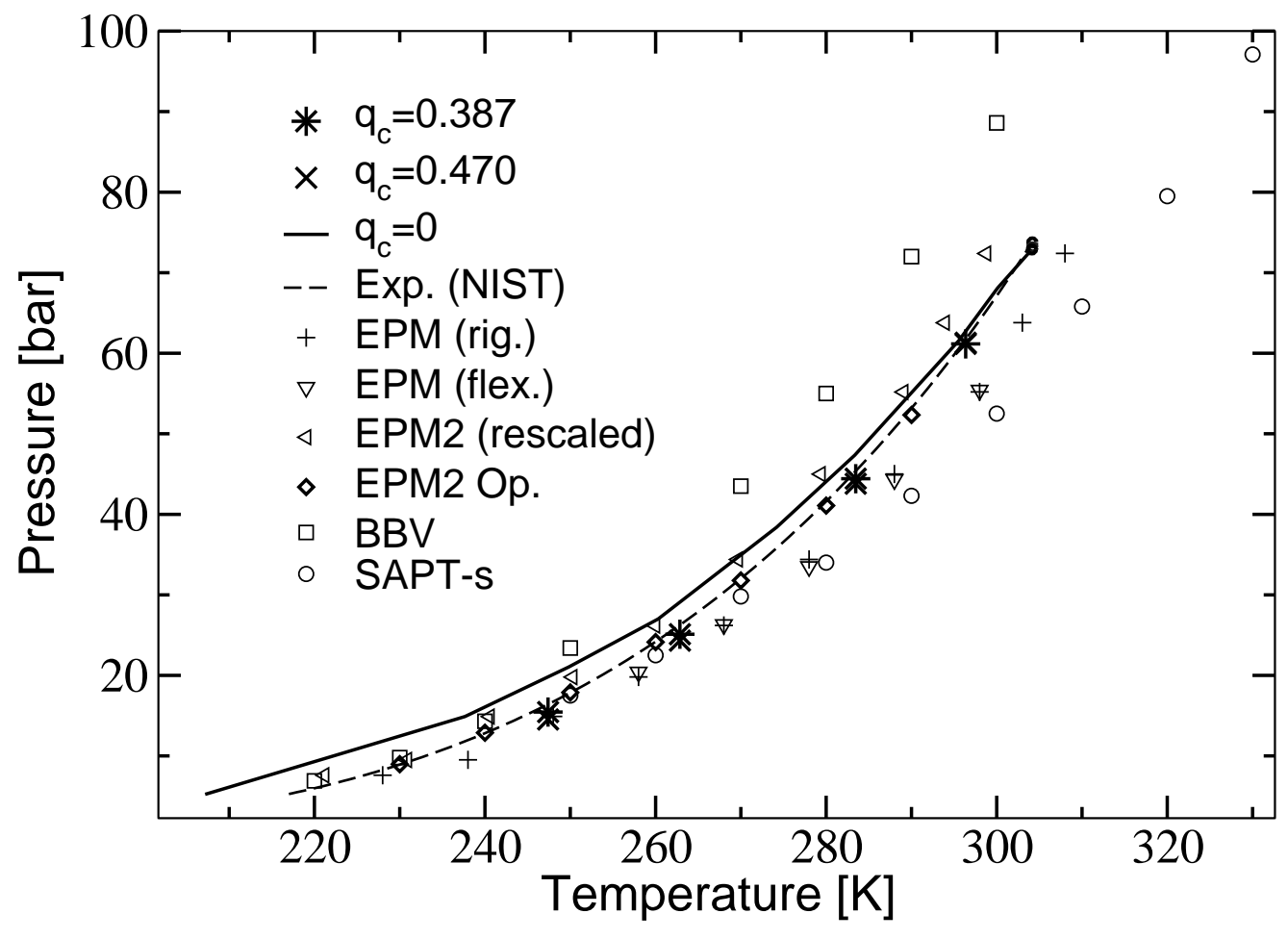

FIG. 7 


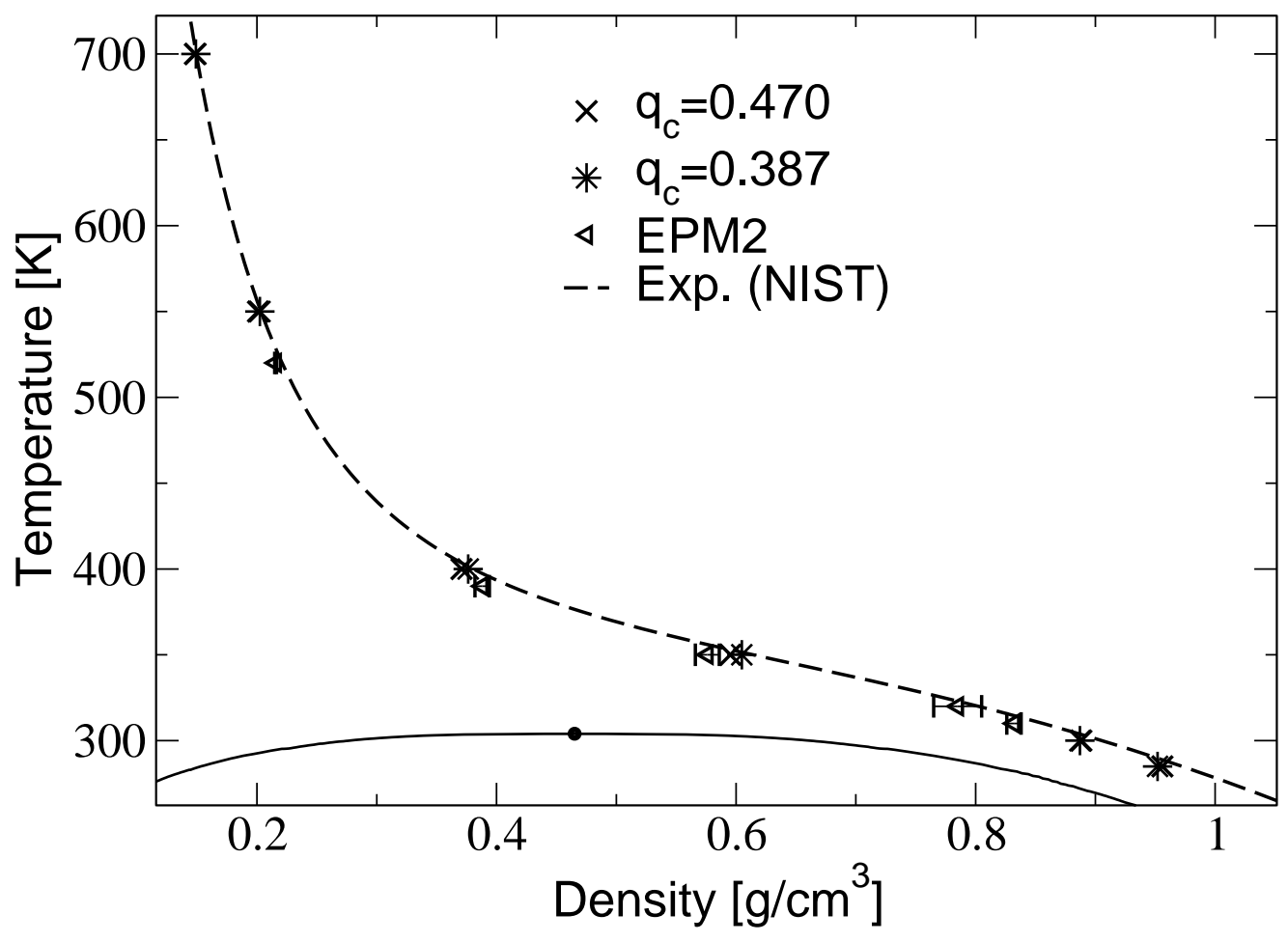

FIG. 8 


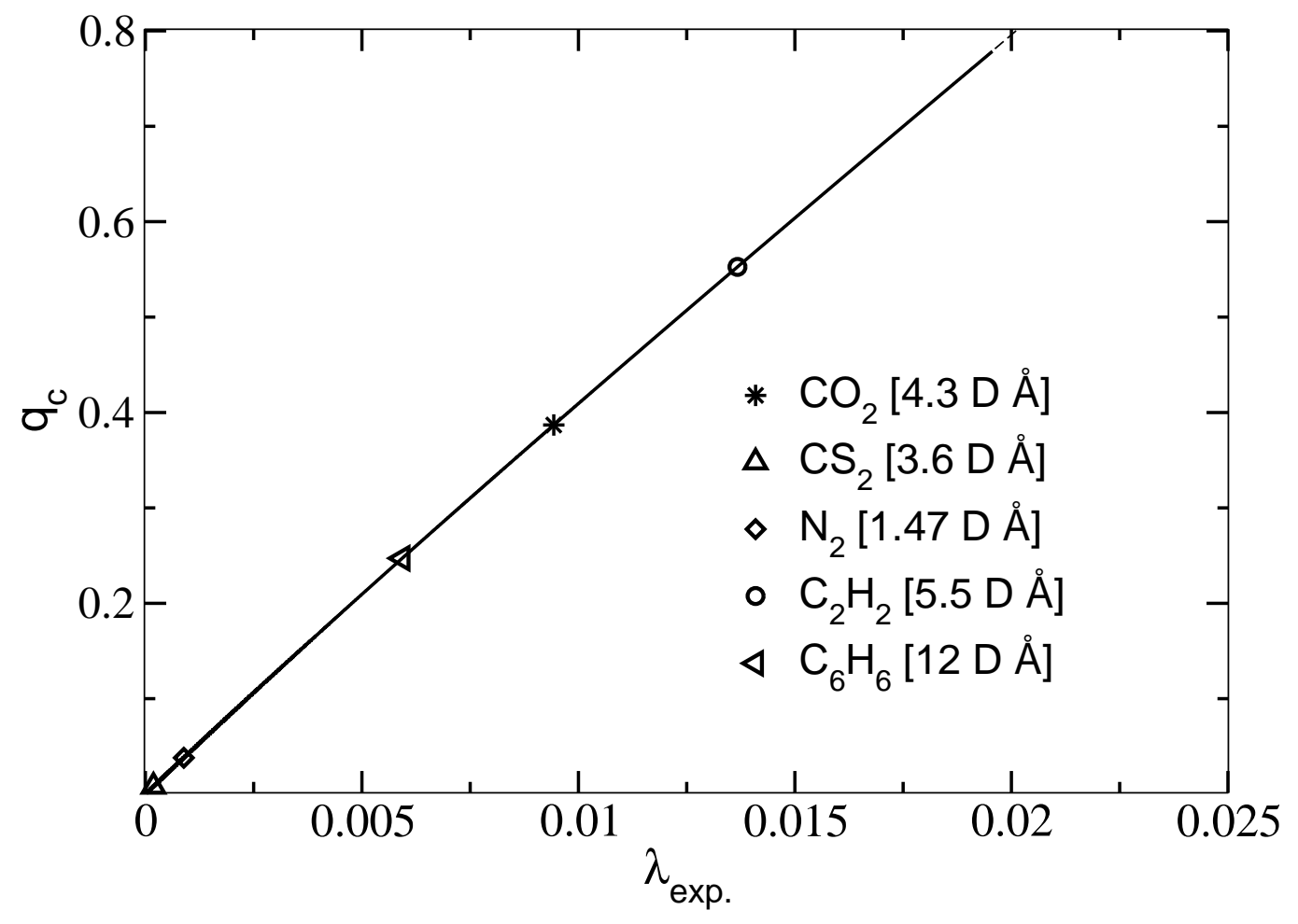

FIG. 9 

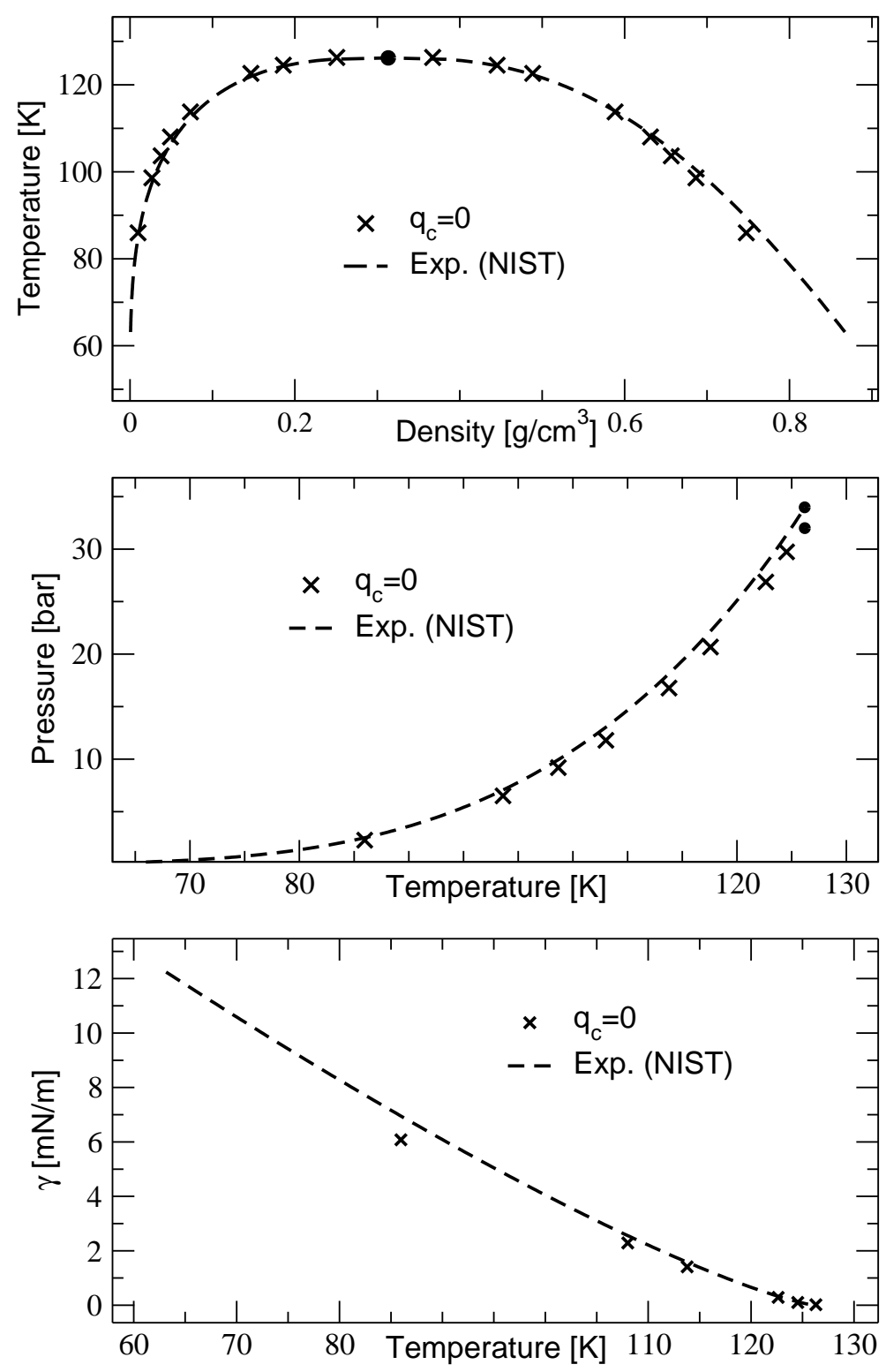

FIG. 10 


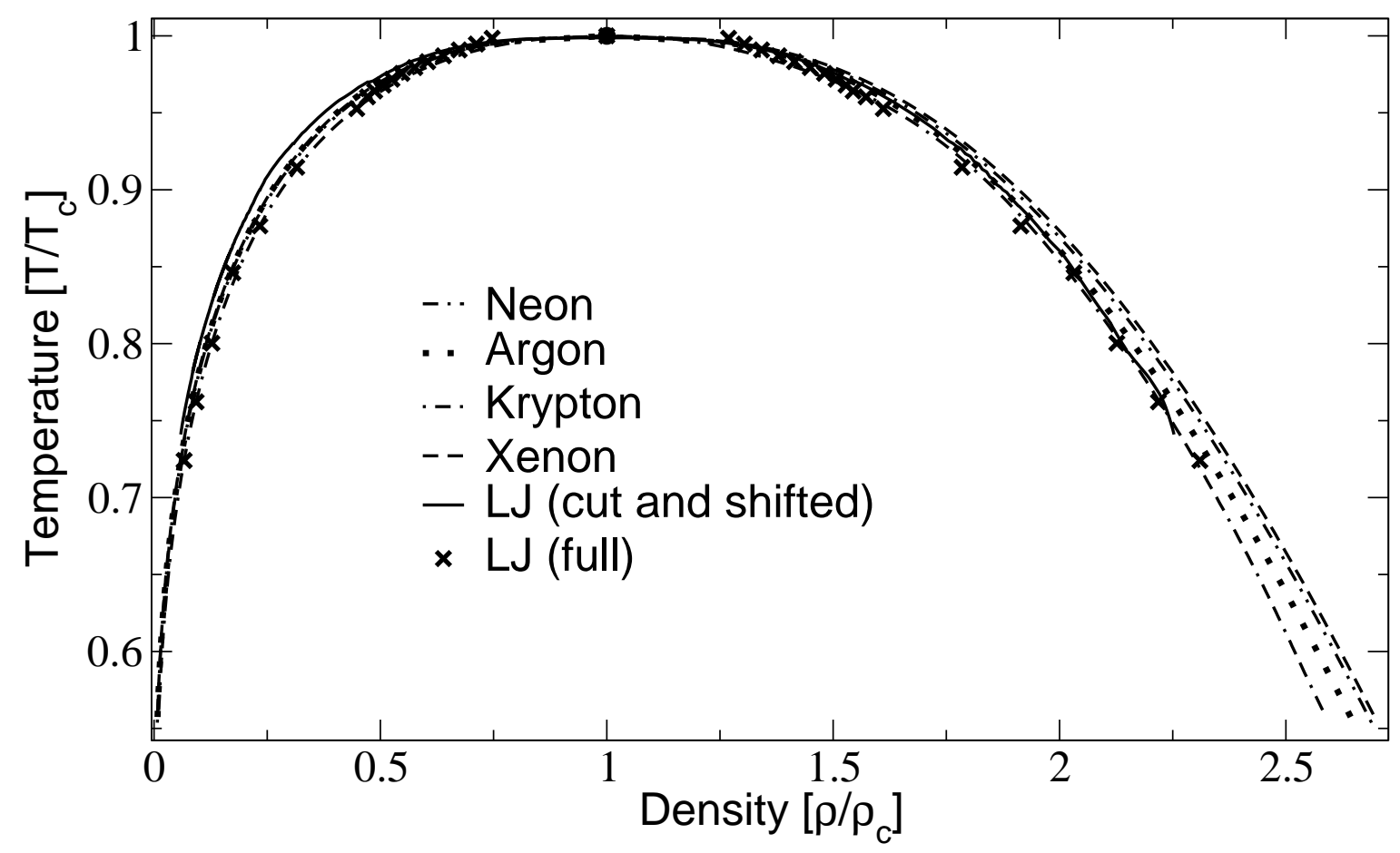

FIG. 11 

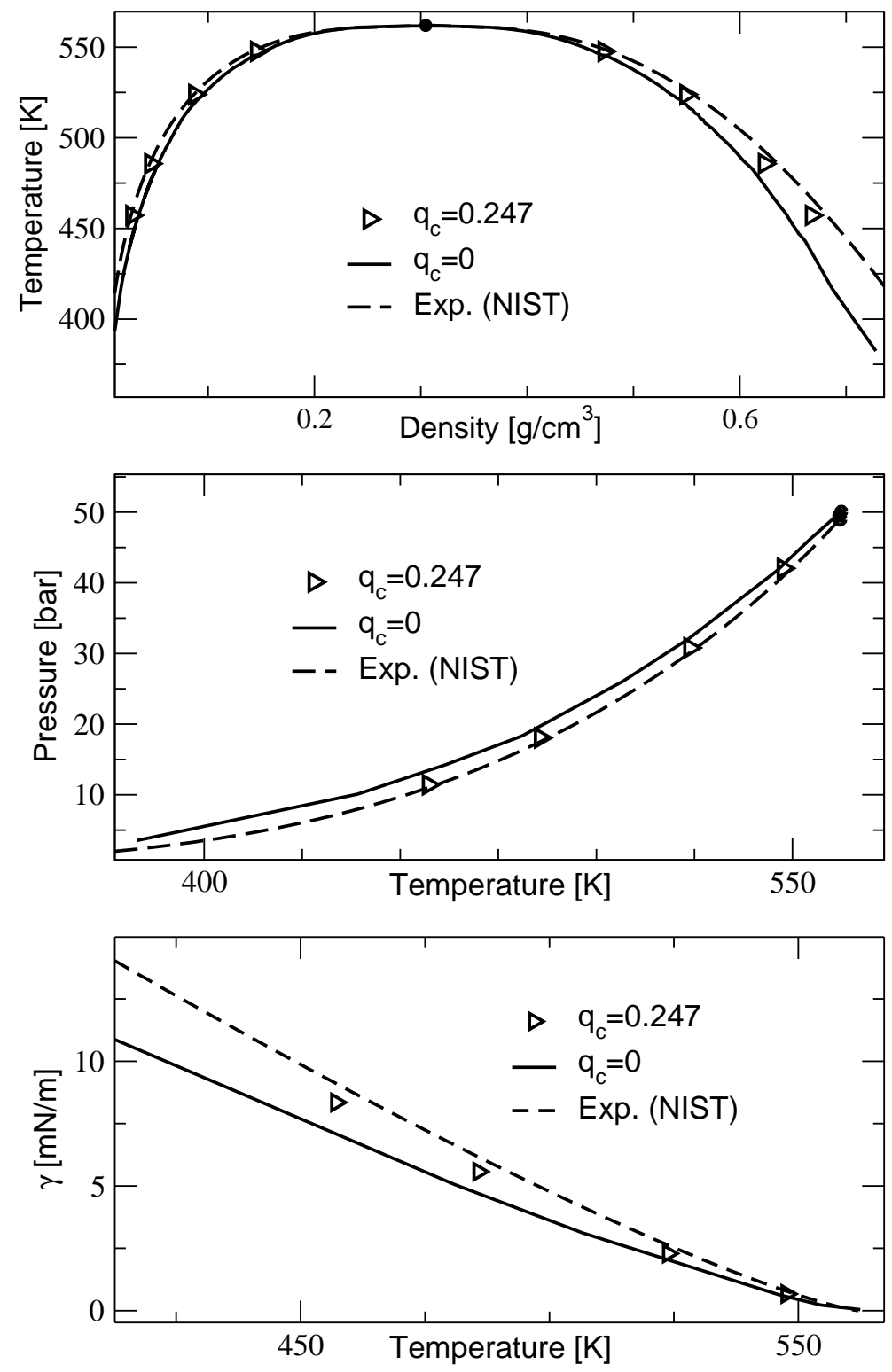

FIG. 12 

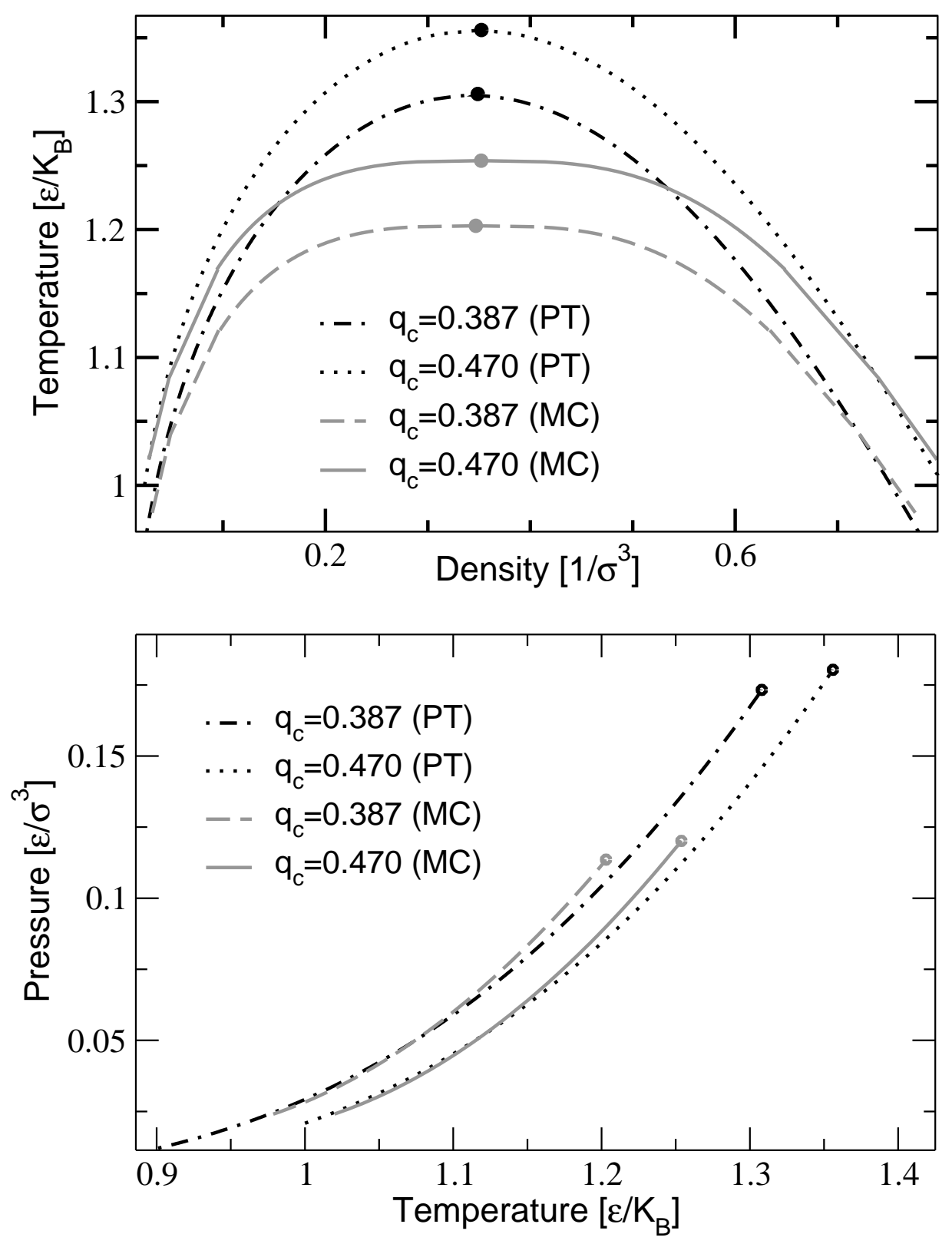

FIG. 13 